\title{
Gas tungsten arc models including the physics of the cathode layer: remaining issues
}

\author{
Isabelle Choquet $^{1}$ (D)
}

Received: 13 February 2017 / Accepted: 14 August 2017 / Published online: 5 September 2017

(C) The Author(s) 2017. This article is an open access publication

\begin{abstract}
A recent review pointed out that the existing models for gas tungsten arc coupling the electrode (a cathode) and the plasma are not yet complete enough. Their strength is to predict with good accuracy either the electric potential or the temperature field in the region delimited by the electrode and the workpiece. Their weakness is their poor ability to predict with good accuracy these two fields at once. However, both of these fields are important since they govern the heat flux to the workpiece through current density and temperature gradient. New developments have been made since then. They mainly concern the approaches addressing the electrode sheath (or space charge layer) that suffered from an underestimation of the arc temperature. These new developments are summarized and discussed, the modelling assumptions are examined, and important modelling issues that remain unexplored are underlined.
\end{abstract}

Keywords (IIW Thesaurus) Refractory cathode . Cathode surface state - Cathode boundary layer - Plasma column $\cdot$ Modelling assumptions

\section{Introduction}

Simulation models for electric arc welding heat sources are useful for, e.g., gaining deeper process understanding,

Recommended for publication by Study Group 212 - The Physics of Welding

Isabelle Choquet

isabelle.choquet@hv.se

1 Department of Engineering Science, University West, 46186 Trollhättan, Sweden doing parametric studies and selecting process windows, providing the local heat input distribution necessary to predict weld material properties, or supporting the development of online process control models. Important outputs they can provide are in particular the heat fluxes from the electric arc (or plasma column) to the workpiece. These heat fluxes are known to be mainly transferred by the current density and the temperature gradients [1]. They include several components, as illustrated by the calculations of Baeva [2] reproduced in Fig. 1. Their prediction necessitates an accurate calculation of the electric potential and the temperature fields in the plasma column; these fields are in turn greatly conditioned by the plasma boundary conditions at the anode surface, and in first place at the cathode surface.

The recent developments made to improve the modelling of the plasma boundary conditions in gas tungsten arc (GTA) mainly concern the cathode plasma interface and approaches addressing the sheath (or space charge layer). The effect of the anode sheath and its potential drop is generally neglected when simulating GTA, e.g., [3]. On the contrary, for simulation models applied to free burning arc in argon, the sheath voltage drop can be set based on experimental measurements, e.g., to $3.5 \mathrm{~V}$ in [4]. Most of the available anode sheath voltage drop measurements were indeed done with free burning arcs or high intensity discharge lamps [5-7]. The available measurements show that the anode sheath voltage drop is lower than the cathode sheath voltage drop, and of same order of magnitude [5]. The shorter cathode to anode distance characteristic of GTA reduces the accessibility to diagnostic and makes the experimental measurements very difficult to carry out. At the anode surface of a high intensity electric arc the anode sheath can either be electropositive or electronegative. It was observed to be electropositive in argon free burning arc at current higher than $50 \mathrm{~A} \mathrm{[5],} \mathrm{whereas} \mathrm{an} \mathrm{electronegative}$ 


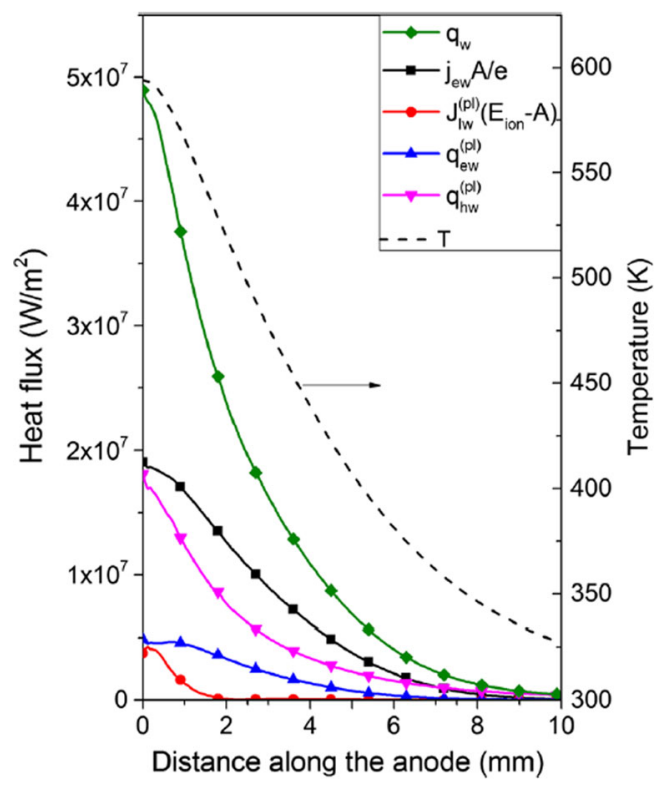

Fig. 1 Temperature (T) along the anode, heat flux to the anode $\left(q_{w}\right)$ and its components from: plasma heavy particles $\left(q_{h w}^{p l}\right)$, plasma electrons $\left(q_{e w}^{p l}\right)$, ion recombination $\left(J_{i w}^{p l}\left(E_{i o n}-A\right)\right)$ and counter diffusing electrons $\left(j_{e w} A / e\right)$ for a 200 A GTA; $5 \mathrm{~mm}$ arc length, water-cooled copper anode. From Baeva [2]

anode sheath was obtained in the presence of helium shielding gas [8]. Based on this knowledge, the anode sheath models for high intensity electric arcs use to assume an electronegative sheath when operated with argon shielding gas, as in e.g. [2]. For other types of shielding gases, and in the presence of metal vapor, measurements are rare so that the type of charge carrier dominating the sheath might also need to be predicted by a model, as further developed in [9]. Very recently, Baeva [2] did evaluate the electronegative sheath voltage drop in an argon GTA with a current of 100$200 \mathrm{~A}$ and an electrode gap of 5-10 mm. The calculations were done applying a non-equilibrium plasma model selfconsistently coupled with a sheath model. The modulus of the anode sheath voltage drop, which was evaluated to be of 0.1-0.2 V, was concluded to be negligible compared to the cathode sheath voltage drop. The electric potential was also solved in the plasma core and the pre-sheath accounting for the diffusion current component in the Ohm law. It showed a negative anode voltage drop of about $3 \mathrm{~V}$ in the pre-sheath [2], while the anode drop was previously attributed to the sheath.

Concerning the plasma boundary conditions at the interface with refractory cathodes, several modelling approaches have been developed. They were recently reviewed within the frame of GTA welding applications [10]. A problem with these approaches was their poor ability to predict both the electric potential and the temperature field in good agreement with experimental measurements [10]. In particular, approaches accounting for a self-consistent modelling of the cathode sheath are known to predict sheath voltage drop and plasma column voltage in good agreement with experimental data over a broad range of current intensity spanning from high intensity discharge lamps to free burning arcs [10, 11]. However, an underestimation of the arc temperature was observed by Baeva et al. [12] applying a model that takes into account thermal and chemical non-equilibrium in the plasma column (model hereinafter referred to as the original fully non-equilibrium (FNE) model). The cathode boundary layer was made of the cathode sheath and the effect of the Knudsen layer was also taken into account. Using a FNE-plasma model the cathode sheath is treated as a boundary layer while the pre-sheath is combined with the plasma column. The underestimation of the arc temperature went hand in hand with an overestimation of the arc attachment area [13]. Restricting this area allowed reducing the temperature discrepancy in the plasma column, but the drawback was the introduction of an adjustable parameter. In fact, the model of [13] does not verify current continuity and energy conservation at the cathode/plasma interface. Recently, the model was significantly improved to match current continuity and energy conservation of the plasma column with the cathode [14]. For this a complete diffusion treatment of particle fluxes, and a generalized Ohm's law were used. These improvements lead to a focusing of the arc and thus an increase of the calculated plasma column temperature (without using an adjustable parameter) [14, 15].

The novel FNE-model [14] was also recently compared to two other numerical approaches describing in different ways the cathode layer coupling cathode and plasma column. The first one is the unified approach developed by Almeida et al. [16]. It is free from any a priori introduction of a boundary layer and consists in a single set of equations valid throughout the cathode layer and the plasma column. Due to its high computational requirements, this model was applied in one-space dimension. The second numerical approach combines cathode layer and plasma column. The plasma column is then modeled with a two-temperature plasma (2T-plasma) model assuming chemical equilibrium (another possible alternative is a local thermal equilibrium or LTE-plasma [11]). The cathode layer is, as in [14], made of a space-charge layer (or sheath), a Knudsen layer, and an ionization layer (or pre-sheath). But the ionization layer is treated as part of the cathode boundary since it cannot be described by a plasma column that assumes chemical equilibrium. These approaches were compared two by two by Benilov et al. [11] computing free burning arc test cases in one-space dimension and two-space dimension (with an hemispherical cathode). At atmospheric pressure and for a current density larger than about $5 \times 10^{7} \mathrm{~A} \mathrm{~m}^{-2}$ the three 
approaches provide calculation results in good agreement since the introduction of a cathode boundary layer and its sub-division in up to three sub-layers is valid in the context of free burning arc [11]. This current density range covers the conditions usually met in GTA applications. However, the electrode geometry is also an important parameter to consider; it is usually much sharper for GTA than for free burning arc.

The novel FNE-model was recently applied by Baeva [2] to a GTA test case with argon shielding gas. The calculation results were compared to experimental measurements of voltage and electron temperature for an inter-electrode gap of $8 \mathrm{~mm}$. The tungsten cathode was doped with lanthana, and the current set to $200 \mathrm{~A}$. The calculated cathode sheath voltage drop was $5 \mathrm{~V}$. The total voltage was defined as the sum of the cathode body, the cathode space-charge sheath, and the arc column voltage. Its calculated value of $14.03 \mathrm{~V}$ is in good agreement with the measured arc voltage of $14 \mathrm{~V}$ (at the arc current of $201.4 \mathrm{~A}$; see [17]). The comparison of the electron temperature calculated in the arc to emission spectroscopy measurements was made along the radial direction at 1 and $4 \mathrm{~mm}$ below the cathode tip. These results, reproduced in Fig. 2, show that thermal equilibrium is reached in the plasma core; thermal non-equilibrium observed in the arc periphery increases with the distance to the arc axis. The electron temperature, $T_{e}$, calculated with the novel FNE-model (denoted "sc" in the figure) is almost everywhere in very good agreement with the measurements. It lies within the experimental error bars except in the cathode tip vicinity where the calculations overestimate the measurements by about $2000 \mathrm{~K}$ on the arc axis at $1 \mathrm{~mm}$ below the tip. The comparison for tungsten cathode doped with thoria is not done; it is well known that this (a)

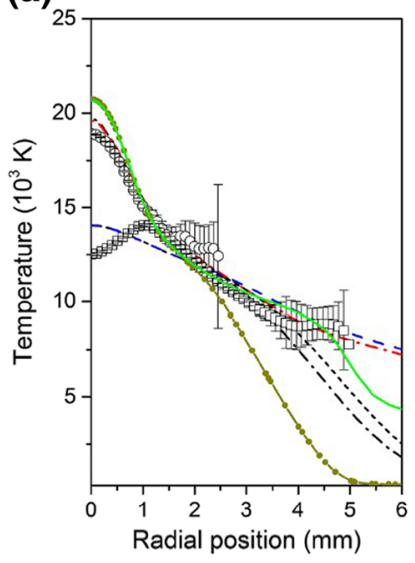

(b)

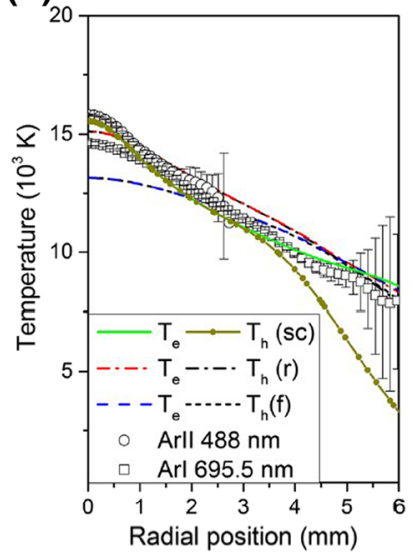

Fig. 2 Temperatures of electrons and heavy particles from FNE models with self-consistent (sc), restricted (r), and free (f) arc attachment in comparison with experimental data along radial lineouts $\mathbf{a} 1 \mathrm{~mm}$ and $\mathbf{b}$ $4 \mathrm{~mm}$ away from the cathode tip. Arc current $200 \mathrm{~A}$, arc length $8 \mathrm{~mm}$. From Baeva [2] dopant is no longer used in manufacturing because of its radioactivity. However it is still of interest for model testing since it has long been studied and is the most documented tungsten cathode dopant [18].

Other recent developments were conducted by Pekker and Hussary [19] and Pekker and Murphy [20] to improve the plasma boundary condition at the interface with a cathode (and an anode). These authors consider a two temperature plasma $[19,20]$, and introduce the metal vapor in the sheath model [20]. Their model is intended to address a broader range of applications than GTA, like the model by Benilov [21, 22]. The approach of Benilov is based on the two-scale description of the cathode layer analyzed by Riemann [23] doing a rigorous kinetic theory of the plasma-sheath transition. The approach of Pekker and Hussary is based on the one-scale description of the cathode layer developed by Godyak and Sternberg [24]. These two approaches differ in several aspects, as underlined in [19]. Four differences are of relevance for GTA at pressure close to atmospheric pressure.

1. Ion collisions in the sheath are assumed (a) negligible in [20-22] and (b) not negligible in [19].

2. The contribution of the thermionic electron number density to the cathode surface electric field is assumed (a) negligible in $[21,22]$ and (b) not negligible in [19, 20].

3. (a) The cathode layer is made of one sub-layer that is the space-charge sheath [19, 20], and (b) the cathode layer is made of two sub-layers that are the space-charge sheath and the ionization layer; these two sub-layers are matched smoothly through the Bohm criterion (Knudsen layer) [21, 22].

4. The contribution of the ion temperature to the Bohm velocity and to the energy balance in the sheath is assumed (a) negligible in $[19,20]$ and (b) not negligible in $[21,22]$.

The models proposed by Pekker et al. were applied to one dimensional test cases in $[19,20]$; to our knowledge they have not yet been applied to GTA. The cathode sheath and Knudsen layer model by Benilov and Marotta were applied to GTA in, e.g., [2, 25]. Thus, the simplified alternatives of 3 ) and 4) have not been used within the frame of GTA, and their validity has not been investigated yet in this context. The different assumptions made in (1) and (2) are discussed thereafter.

On the other hand, GTA studies were recently conducted with a simple LTE-plasma column model dividing the cathode layer in three sub-layers [25, 26]. It implies that the deviations from thermal and chemical equilibrium are both localized in a cathode sub-layer (the ionization layer). The model is also self-consistent and based on fundamental 
physical principles, e.g., charge and energy conservation. It involves the cathode sheath and the Knudsen layer model of Benilov and Marotta [21, 22], and a cathode pre-sheath model mainly based on the developments by Cayla et al. $[27,28]$. This cathode layer model is suited to a local resolution of the sheath along the cathode surface, i.e., the sheath properties are not assumed uniform in space [10]. The model (see Appendix A) was applied to compute a 200 $A$ and stationary argon arc produced by a thoriated tungsten electrode, with a conical tip of angle $60^{\circ}$, on a water cooled workpiece. The electrode to workpiece distance was $5 \mathrm{~mm}$. This test case, which is described in further details in Appendix B, was retained since measurements of both the cathode surface temperature and the electron temperature in the arc were available [29, 30], while temperature prediction was the weakness of the modelling approach. Calculation results are plotted in Figs. 3 and 4. Figure 3 compares the calculated cathode surface temperature with the experimental measurements of Haidar and Farmer [30]. At the tip end (located at distance zero in Fig. 3) the calculated cathode surface temperature underestimates the experimental measurements, while towards the tip shoulder at about $2 \times 10^{-3} m$ it overestimates the measurements. The maximum discrepancy is then of the order of $400 \mathrm{~K}$. Figure 4 compares the plasma temperature calculated along the arc symmetry axis with the electron temperature measurements of Haddad and Farmer [29]. It can be seen that the underestimation of the plasma temperature is significantly pronounced; it reaches almost $8000 \mathrm{~K}$ at a distance $z=$ $10^{-3} \mathrm{~mm}$ below the cathode tip end. A natural set of questions is then: why do these calculations underestimate to a large extend the GTA arc temperature measured experimentally? Which modelling assumption is justified and which one should be questioned?

The modelling assumptions are listed and critically examined for the three following regions: the solid cathode (Section 2), the cathode layer (Section 3), and the thermal

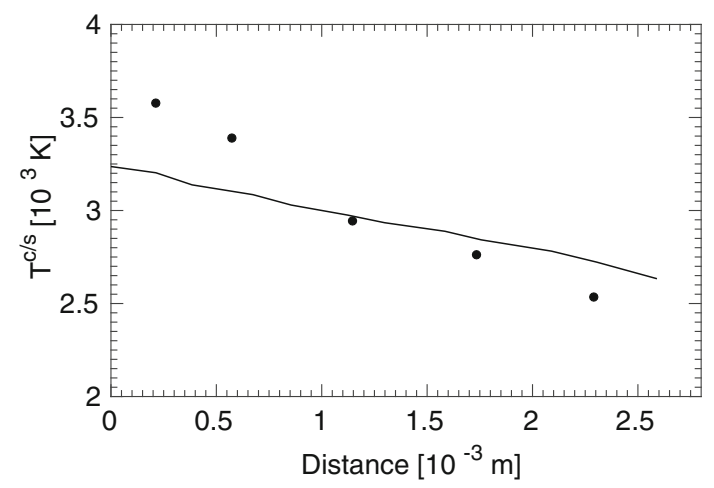

Fig. 3 Temperature along the cathode surface as function of the distance from the tip center. (-): calculation results, and $(\bullet)$ : experimental measurements of Haidar and Farmer [30] at 200 A

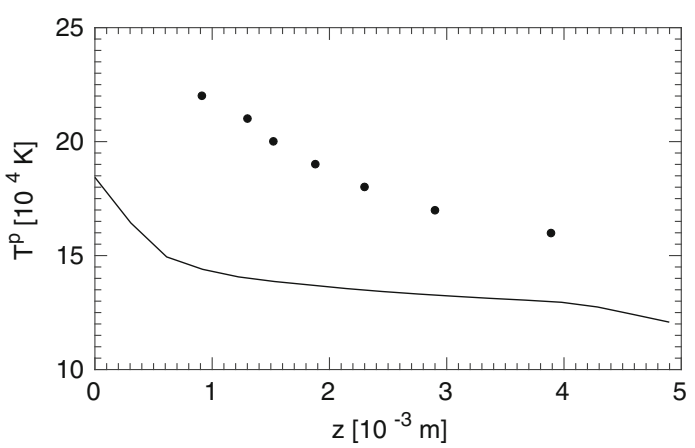

Fig. 4 Temperature along the arc axis as function of the distance

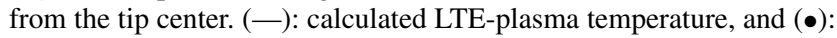
electron temperature measured by Haddad and Farmer [29] at $200 \mathrm{~A}$

plasma (Section 4). The paper is structured starting from the LTE-model $[25,26]$ used above to exemplify the problem (Figs. 3 and 4). Numerical results are presented in the sequel to illustrate the effect of some modelling assumptions. Modelling assumptions specific to the FNE-approach by Baeva et al. [11, 14], and to the approach developed by Pekker at al. [19, 20], are also addressed. Finally, important modelling issues that remain unexplored are discussed in conclusion.

For clarity and completeness the model used to do the computations is given in Appendix A, while the numerical test case is defined in Appendix B. In this model, the temperature distribution in the cathode is self-consistently calculated, while the surface temperature of the anode is not calculated; it is assumed to be given by a boundary condition for water-cooled anode (further details are provided in Appendix B, Table 3). For the GTA test case calculated with a FNE-approach by Baeva [2], a water-cooled anode with negligible vaporization and melting was also assumed but the anode temperature was calculated (see Fig. 1). The water-cooled anode condition is useful to perform spectroscopic measurements and test the models. When moving from the laboratory to the workshop, the condition at the anode is very different. The anode spot temperature can in practice be very high, which may lead to significant evaporation of the anode material and change the arc dynamics [31]. This issue is not addressed here.

\section{Cathode-modelling assumptions}

When considering the cathode sheath, the cathode model (cathode surface included) uses to be based on the following assumptions, as in [12] and [14]:

(A0) Joule heating in the cathode body can be significant,

(A1a) radiative heating of the cathode surface is negligible compared to radiative cooling,

(A2) secondary electron emission is negligible, 
(A3a) electron emission can be modeled by the Richardson Dushman emission law supplemented with Schottky correction,

(A4a) the cathode is homogeneous with a uniform physical state.

Assumption (A0) can be verified since GTA welding applications cover a wide current range. At low current, Joule heat generation in the cathode body is known to be negligible. Above some current threshold $I^{\star}$ this heat source term is important to model since it has an effect on e.g. the cathode surface temperature, as shown by Benilov and Cunha [32]. These authors modeled cylindrical tungsten cathodes in argon plasma for various configurations, and current ranging from 1 to $500 \mathrm{~A}$. From their results, it can be seen that the current threshold $I^{\star}$ depends on several parameters such as the electrode radius $R$ (varied from 0.3 to 1.0 $\mathrm{mm}$ in [32]) and the tip geometry (hemispherical, or flat in [32]). For the set of configurations studied in [32] the current threshold $I^{\star}$ was observed to vary from $26 \mathrm{~A}$ (when $R=$ $0.3 \mathrm{~mm}$ ) to $274 \mathrm{~A}$ (when $R=1.0 \mathrm{~mm}$ ). It should be noticed that parameters other than $R$ (such as the argon pressure and the inter-electrode distance) were simultaneously varied in [32], and to our knowledge the exact dependence of $I^{\star}$ is not established yet. Thus, apart from few specific configurations and process parameters, the value of $I^{\star}$ is generally not know. A systematic modelling of Joule heat generation in the cathode body (as done in, e.g., [2, 25], and in Eq. 7 of Appendix A) allows covering the wide range of GTA welding applications without needing to know whether the process current is below or above the current threshold $I^{\star}$.

The radiation heat flux emitted locally by the cathode surface is generally expressed using the gray body model. The radiation heat flux reaching the cathode surface includes radiation from the plasma column and from the surrounding surfaces, in particular the anode surface. The radiation from the anode surface that reaches the cathode tip was evaluated in [25] for a water cooled copper anode. It is several orders of magnitude lower than the radiation from the plasma column, and thus negligible. The incident radiation heat flux reaching the cathode surface while emitted from the plasma column can be calculated using the view factor method, as in [25].

The fraction of incident radiation that is locally reflected by the cathode surface is usually ignored. Reflection modelling is a difficult issue that would need knowing, e.g., the local fraction of diffuse and specular reflection. The reflected heat has no direct effect on a standard GTA cathode surface (due to convexity); however, it could have an effect on the plasma temperature. This effect, which should be globally small compared to other plasma source terms such as Joule heating, still needs to be quantified locally.
The fraction of incident radiation that is locally absorbed by the cathode is governed by the surface absorptivity. Larrabee [33] measured the emissivity of incandescent tungsten as function of both the wavelength and the temperature for surface temperatures ranging from 1600 to $2400 \mathrm{~K}$. Applying Kirchhoff's law of radiation, the emissivity determined in [33] leads to an absorptivity ranging from 0.41 to 0.485 [25]. Nomerovannaya et al. [34] measured the absorptivity of tungsten monocrystals over a broader range of wavelengths. For the range corresponding to incandescent tungsten, their results show an absorptivity of about 0.5 . Thus, the emissivity of incandescent tungsten is not negligible, and assumption ( $A 1 a)$ might be questioned. The effect of cathode surface heating by plasma radiation was investigated in [25]. The radiation heat fluxes calculated along the cathode surface assuming that

(A1b) radiative heating of the cathode surface is not negligible compared to radiative cooling,

are plotted in Fig. 5 for a tungsten absorptivity set to 0.5 (i.e. pure tungsten $[33,34])$. These results show that the radiative heating and cooling of the cathode surface are of same order of magnitude; assumption (A1b) thus prevails in this case. The electrodes used in GTA welding are commonly made of tungsten activated with a few percent in weight rare earth oxide for stability purpose and to facilitate arc ignition [35]. The presence of rare earth oxide might modify the emissivity of doped tungsten compared to pure tungsten. This issue is poorly documented. There is thus a need for new experimental investigations to determine the effect of the presence of rare earth oxide on the surface emissivity (or absorptivity) of tungsten.

Assuming (A1b) rather than (A1a) has a consequence on the computational time, which is larger with (A1b) since the view factor method is computationally demanding. A

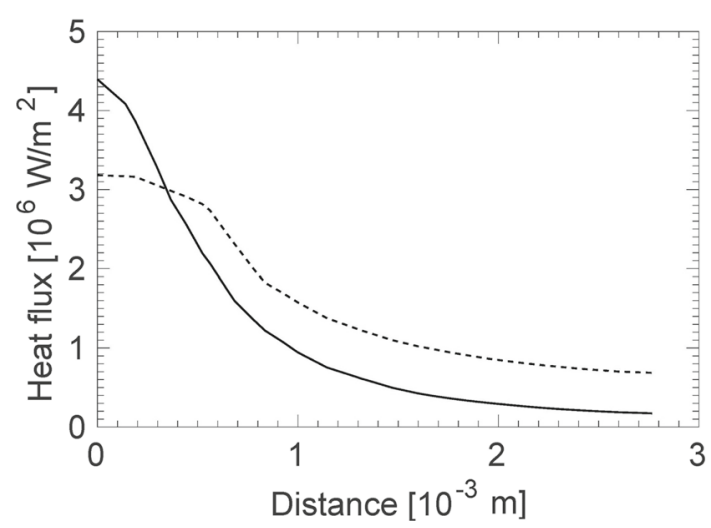

Fig. $5(-, \cdots)$ absorbed and emitted heat flux $\left(q_{\text {rad }}^{a b s}, q_{\text {rad }}^{\text {em }}\right)$ along the cathode surface as functions of the distance from the tip center Non-homogeneous cathode model, assumption (A1b) 
second consequence concerns the cathode and the arc properties. It was shown in [25] that if the cathode layer is modeled only over the electrically conducting part of the cathode surface, using assumption (A1a) rather than (A1b) has only little effect on the cathode surface properties and on the arc properties. The reason is the low value of emitted and absorbed radiative heat fluxes compared to other heat fluxes transported by, e.g., electrons and ions, in the central part of arc attachment area (see Section 3.1). At the transition region between electrically conducting and non-conducting cathode surface, radiative emission and absorption are not negligible compared to the other heat fluxes. Assumptions (A1b) could thus have an effect on the size of the arc attachment, and in turn on the cathode and the arc properties. To our knowledge, computations assuming (A1b) while modelling the cathode layer all over the cathode surface have not yet been made. Further investigations are thus needed.

It is known that for a tungsten cathode secondary electron emission is not negligible compared to electron emission when the current density is, in absolute value, less than $5 \times 10^{6} \mathrm{~A} / \mathrm{m}^{2}[27,28]$. This threshold value can be met for high intensity discharge lamp applications. However, it is expected to be below the current density range of GTA. It was checked in [26] that for the GTA test cases investigated experimentally in $[29,30]$ with a W-2\%wt $\mathrm{ThO}_{2}$ cathode, secondary emission is negligible compared to electron emission, so that assumption (A2) is satisfied. It should be noticed that, for computational purposes, secondary electron emission might however be convenient to consider. It was observed to provide numerical stability when initializing GTA calculations from a low current $(5 \mathrm{~A})$ and ramping up to the operating GTA current [26].

The Richardson Dushman emission law supplemented with Schottky correction is known to be valid for thermionic emission and field enhanced thermionic (or thermofield) emission in the presence of a small cathode surface electric field. There is little information available about how small the surface electric field should be to apply Schottky correction within its domain of validity. The answer depends on several parameters, e.g., the material work function and the surface temperature. An inappropriate emission law would have an effect on the temperature fields computed at the cathode surface and in the plasma column. He [36, 37] did delimit, as function of the surface electric field, emission zones in which modelling assumptions hold. However, this was made for conditions that differ from usual GTA conditions (a cathode surface temperature of $1000 \mathrm{~K}$ and pure tungsten). Besides, Coulombe and Meunier [38] did question the validity of Schottky correction for a material work function of 2,6 eV (which is close to the work function of some rare earth oxides). These authors did show that in that case the Richardson Dushman emission law supplemented with Schottky correction can deviate from the Murphy Good emission law. The Murphy Good emission law is known to encompass the Richardson Dushman emission law and to be valid over a broader range of cathode surface electric field. A drawback is the much larger computational time it requires [26]. The effect of electron emission law was investigated in [26] replacing (A3a) with the following assumption:

(A3b) electron emission should be modeled by the Murphy Good emission law.

It was checked in [26] that for the GTA test cases investigated experimentally by Haddad and Farmer [29] and Haidar and Farmer [30] with thoria electron emitters, the Richardson Dushman emission law supplemented with Schottky correction is still used within its domain of validity. It does not deviate from the Murphy Good emission law. Assumption (A3a) is thus verified.

For a newly made GTA electrode, it can be assumed that the activator islands (few percent in weight) are almost uniformly distributed throughout the volume of the tungsten matrix. The electrode can then be described at the macroscopic scale by a single physical state (called here state I), and assumption (A4a) is satisfied. In the physical state (I), the rare earth electron emitters are thus scattered over the cathode surface. For W-2\%wt $\mathrm{ThO}_{2}$, they occupy a surface fraction of the order of $5 \%$, as evaluated by Yamamoto et al. [39]. In this state the electron emitters are believed to be the low work function activator islands rather than the large work function tungsten matrix. It implies that in state (I) only a small fraction of the electrode surface might contribute to electron emission.

When a GTA electrode interacts with an electric arc, the cathode temperature can locally reach the melting temperature of the rare earth activator; this temperature is below the melting temperature of the tungsten matrix (see Table 1). The melted activators then diffuse along the tungsten grain boundaries, towards the cathode surface, due to the temperature gradients. This phenomenon, discovered by Langmuir with tungsten filaments [44], was further investigated via studies on e.g. electrode erosion $[18,35$, 45-47]. It is known that the rare earth oxides do not all behave in the same way during their diffusion. Thoria does not react chemically with the tungsten matrix, while the other rare earth oxides do [18]. The type of chemical reactions taking place and how these reactions control the diffusion process leading to the formation of a new physical state (II) on the cathode surface is very poorly documented. It would be useful to know if the diffusion process is controlled by the melting of the rare earth oxide, or by its chemical reaction(s) with the tungsten matrix. A better knowledge about the processes involved in the formation of the physical state (II) would allow modeling this phenomenon. 


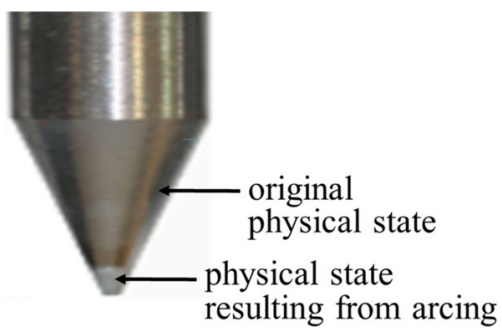

Fig. $6 \mathrm{~W}-2 \% \mathrm{wtThO} \mathrm{T}_{2}$ electrode after arcing

When the new physical state (II) is formed, as a result of the cathode interaction with the arc, the assumption (A4a) is no longer valid all over the cathode surface, in particular at the cathode tip (see Fig. 6). In the physical state (II), the rare earth electron emitters form an almost uniform layer over the surface. The presence of more than one physical state results in a local change of the cathode ability to emit electrons. The emission law then depends on both the local cathode surface temperature and the local physical state. The local physical state is thus expected to have an effect on the arc attachment and the temperature fields at the cathode surface and in the plasma column.

For some operating conditions (depending on, e.g., current intensity, process duration, shielding gas, etc.), the cathode surface temperature might also reach the vaporization temperature of the electron emitters constituting the surface layer in state (II). Then, more than two physical states can be formed, as further developed in, e.g., [25, 35].

The cathode models used up to recently for GTA assume (A4a). They define the flux of emitted electrons taking into account the local cathode surface temperature and neglecting the local physical state. Javidi Shirvan et al. [25, 26] did extend the modelling of the flux of emitted electrons to take into account, at a first level, the local physical state of the cathode surface (assumption (A4b)). This development is based on the following physical criteria and limitations:

(A4b.1) The cathode temperature is assumed to remain below the vaporization temperature, $T_{v}^{c}$, of the rare earth electron emitters diffused to the surface (so a maximum number of two states can be present).

(A4b.2) The percentage in weight of activator in the tungsten matrix in physical state (I), which uses to be of the order of 1 to $2 \%$ in weight, is small enough to assume in a first approximation that electron emission is negligible in the physical state (I) compared to the physical state (II).

(A4b.3) Physical state (II) starts where the characteristic temperature, $T_{d}^{c}$, controlling the diffusion process is reached. The surface of this region is supposed to be fully covered by a layer of rare earth emitter as a result of diffusion. In other words, in physical

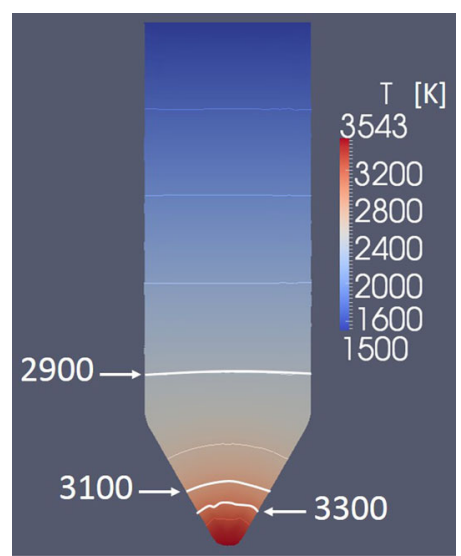

Fig. 7 Temperature field calculated in the cathode; assumption (A4b) and LTE-plasma approach

state (II), electron emission can take place from any part of the cathode surface, and the work function for electron emission is the work function of the diffused rare earth emitter constituting the surface layer.

The temperature calculated in the cathode domain for the test case of Appendix B and doing assumption (A4b) is plotted in Fig. 7. In this test case, the activator is thoria, thus $T_{d}^{c}=T_{m}\left(\mathrm{ThO}_{2}\right)$ and $T_{v}^{c}=T_{v}\left(\mathrm{ThO}_{2}\right)$. Thoria properties used for the computations are given in Table 1. It can be checked in Fig. 7 that the maximum cathode surface temperature $(3543 \mathrm{~K})$ is everywhere lower than the vaporization temperature of the activator $(4670 \mathrm{~K})$, implying that the modelling assumption (A4b.1) is apparently satisfied. The cathode surface temperature calculated with the assumption (A4a) and (A4b) are plotted in Fig. 8. The experimental measurements of Haidar and Farmer [30] are also reported in this figure. It can be seen that assumption (A4b), which takes into account a first effect of the arc on the physical state of the cathode, leads to calculation results in better agreement with the experimental measurements than (A4a).

However, assumption (A4b) still makes several simplifications; their validity needs also to be further explored. For instance it has not yet been checked whether the role played by electron emission in the physical state (I) is actually negligible as assumed in (A4b.2). Vaporization of the cathode surface in physical state (II) can also take place while maintaining the surface layer of rare earth electron emitters, as long as depletion by vaporization can be balanced

Table 1 Properties of thermionic cathode emitters [35, 40-43]

\begin{tabular}{lllll}
\hline & $\lambda_{R} A_{0}\left[A \cdot m^{-2} \cdot K^{-2}\right]$ & $\phi[\mathrm{eV}]$ & $T_{m}[\mathrm{~K}]$ & $T_{v}[\mathrm{~K}]$ \\
\hline $\mathrm{W}$ & $8 \times 10^{5}$ & 4.5 & 3653 & 6203 \\
$\mathrm{ThO}_{2}$ & $3 \times 10^{4}$ & 2.6 & 3323 & 4670 \\
\hline
\end{tabular}




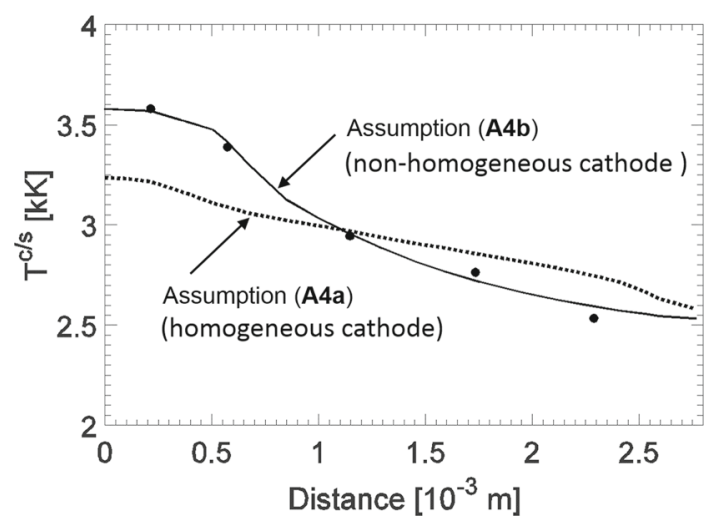

Fig. 8 Temperature along the cathode surface as a function of the distance from the tip center: experimental measurements by Haidar and Farmer [30] (•), and calculation results with the LTE-plasma approach

by diffusion from the cathode bulk [26]. The criterion of local vapor pressure, which is not considered in assumption (A4b.1), is also important to explore. It would require modelling the effect of the metal vapor, first of all on the cathode layer. Metal vapor emitted from the cathode is expected to have an effect on the energy transfer through the cathode layer, on the arc attachment and on the temperature and current density fields. A first model accounting for the effect of metal vapor on the cathode sheath was introduced by Pekker and Murphy [20]. It was applied to pure tungsten cathode (and to a copper anode) considering a 1-dimensional thermal plasma. Vaporized tungsten was observed to have little effect on the cathode sheath (contrary to copper in the anode sheath). The effect of the rare earth oxides constituting the cathode surface layer in physical state (II) on the cathode sheath remains to be investigated. Also, after some operating time more physical states are known to be formed [18, 35, 45-47]. Their modelling has not been addressed yet. Finally, a fundamental problem is the incomplete knowledge concerning the diffusion mechanisms of cathode activators. The diffusion mechanisms cause the local modifications of the cathode physical state during arcing, and their characteristic thresholds are important parameters for modelling. The model parameters characterizing cathode physical state transitions are well documented for thorium dioxide since this material has been studied for a long time [18]. But nowadays, new legislations force the use of thorium-free cathode, and the diffusion mechanisms of rare earth metal oxides other than thorium are still poorly known.

\section{Cathode layer-modelling assumptions}

The cathode layer model is used to set the boundary conditions coupling cathode and plasma column, as further detailed in [26] for a LTE-plasma and in [14] for a FNEplasma. For this, it has to provide locally along the surface the following data: the cathode surface temperature, the cathode sheath voltage drop and the electron temperature at the plasma-sheath boundary. Based on the generalized Bohm criterion for sheath formation developed by Riemann [23], the cathode layer includes the three sub-layers sketched in Fig. 9: the cathode sheath, the Knudsen layer and the cathode pre-sheath (i.e., the ionization layer). When assuming a plasma column in chemical equilibrium (either LTE- or 2T-plasma), these three sub-layers are included in the cathode boundary layer model, as in $[10,26]$. When assuming a plasma column in chemical non-equilibrium (FNE-plasma), the cathode boundary layer model reduces to the cathode sheath and the Knudsen layer since the presheath is combined with the plasma column, as in $[2,14]$. To model the cathode layer, it is usually assumed that

(A5) the cathode sheath approach is valid;

(A6) each sub-layer of the cathode boundary layer can be modelled as a boundary layer;

(A7) the thickness of the cathode boundary layer is uniform along the cathode surface.

Assumption (A5) requires first that ionization is negligible in the sheath, and second that the electrons are in equilibrium with the electric field [24]. Consider the first requirement. The thickness of the sub-layer is of the order of the Debye length $\left(\lambda_{D} \approx 10^{-8} \mathrm{~m}\right)$ for the cathode sheath, of the smallest collisional mean free path $\left(\lambda_{c} \approx 10^{-7} \mathrm{~m}\right)$ for the Knudsen layer, and the recombination length $\left(\lambda_{r} \approx 10^{-5} \mathrm{~m}\right)$ for the cathode pre-sheath [10]. The values in bracket were obtained by Benilov and Marotta for an argon plasma with a number density of about $10^{23} \mathrm{~m}^{-3}$ and temperature of the order of $10000 \mathrm{~K}$ [21]. It corresponds to the order of magnitude of the temperature calculated in the cathode sheath vicinity with the test case of Appendix B $\left(T_{e} \approx 12000 \mathrm{~K}\right.$

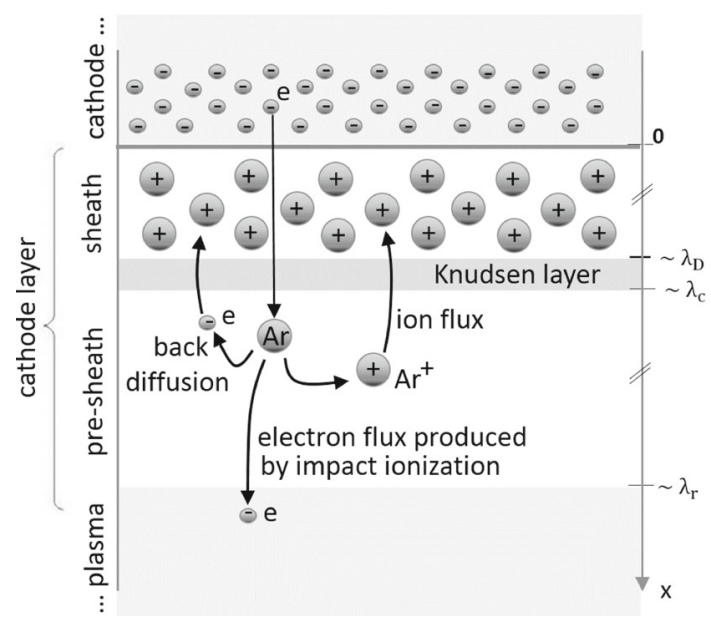

Fig. 9 Sketch of sub-regions in a GTA cathode layer [10] 
and $T_{h} \approx 3500 \mathrm{~K}$, see Figs. 8 and 10 at the cathode tip). For the GTA test case calculated in [2] with the novel FNEapproach, the temperatures obtained in the cathode sheath vicinity (on the arc symmetry axis) are $T_{e} \approx 18000 \mathrm{~K}$ and $T_{h} \approx 3000 \mathrm{~K}$, leading to $\lambda_{D} \approx 10^{-7} \mathrm{~m}$ and $\lambda_{r} \approx 10^{-5} \mathrm{~m}$. As, in each case, $\lambda_{D}<<\lambda_{r}$, the first requirement is verified. The second requirement is satisfied if the fraction of back-diffusion electrons entering the sheath is very small. Referring to Eq. 17 in Appendix this condition is verified if $\exp \left(-\left(e U^{s}\right) /\left(k_{b} T_{e}^{p s / s}\right)\right)<<1$. This quantity was evaluated along the cathode surface for the GTA test case described in Appendix B with assumption (A4b). Figure 10 shows the calculation results plotted in the arc attachment region. It can be seen that the second requirement for applying a sheath approach is also satisfied. However, this check is only partial. As these calculations were done using simply a LTE-plasma model for the plasma column, the criterion could not be calculated all over the cathode surface. Using a 2T- or a FNE-plasma model the validity of the condition $\exp \left(-\left(e U^{s}\right) /\left(k_{b} T_{e}^{p s / s}\right)\right)<<1$ could also be checked on the cathode surface outside the arc attachment region.

If, as for free burning arcs, the arc attachment radius $r_{a r c} \approx 10^{-3} \mathrm{~m}$ is used to define a characteristic length, then $\lambda_{r}<<r_{a r c}$. It implies that assumption (A6) is verified, as checked for free burning arcs in [11]. For GTA applications, the cathode tip end radius is one order of magnitude less than the arc attachment radius, $r_{t i p} \approx 10^{-4} \mathrm{~m}$. If $r_{t i p}$ is used to define the characteristic length, then $\lambda_{r}<r_{t i p}$ and assumption (A6) should still be satisfied. But this has not been checked yet. The problem might be more complex for GTA since the conditions cannot be assumed uniform along the cathode surface. This point is further developed below when discussing assumption (A7).

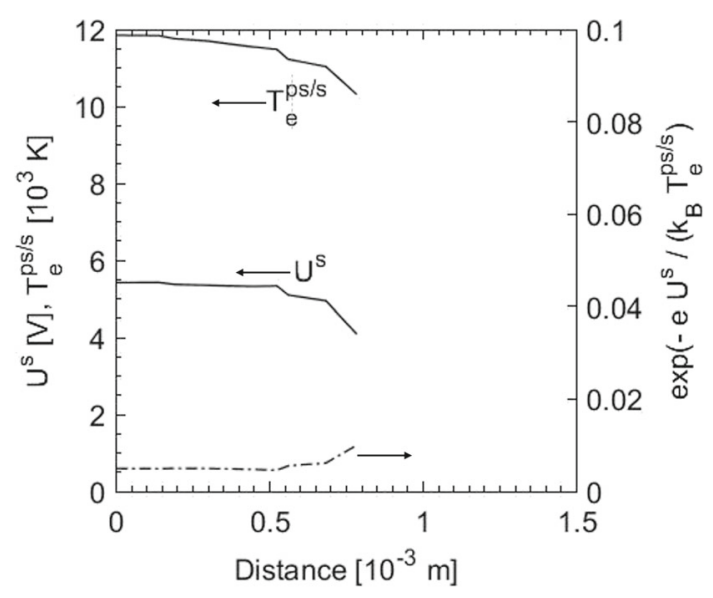

Fig. 10 Cathode sheath voltage drop, $U^{s}$, electron temperature at the pre-sheath boundary on the sheath side, $T_{e}^{p s / s}$, and criterion for validity of the one-scale cathode layer approach calculated as functions of the distance from the cathode tip center
For a FNE-plasma approach, the plasma column is in thermal and chemical non-equilibrium; the modelling of the pre-sheath is then included in the (3-dimensional) plasma column rather than the (1- or 0 -dimensional) cathode boundary layer. In this case the cathode boundary sub-layers are only the sheath and the Knudsen layer, and assumption (A7) is clearly satisfied. The cathode boundary layer is then modelled as a 0-dimensional interface (see [2, 14]). For a LTEas well as a 2T-plasma model the cathode boundary layer includes sheath, Knudsen layer and also pre-sheath. The thickness of the cathode boundary layer is then mainly governed by the thickness of the pre-sheath. This thickness can be evaluated with the ionization length calculated for an atmospheric-pressure argon plasma in [48] as a function of the electron $T_{e}$ and heavy particle temperatures $T_{h}$. These temperatures were calculated at the ionization layer boundary on the sheath side in [26] for the GTA test case of Appendix B and the assumption (A4b). At the cathode tip end it gives $T_{e}=11850 \mathrm{~K}$ and $T_{h}=3543 \mathrm{~K}$, and at a distance of $1 \mathrm{~mm}$ from the tip $T_{e}=8100 \mathrm{~K}$ and $T_{h}=3050 \mathrm{~K}$. According to Fig. 2 in [48], the ionization length is $95 \mu \mathrm{m}$ and $300 \mu \mathrm{m}$, respectively. Then, the thickness of the ionization layer is not uniform along the electrically conducting part of the cathode surface. When applied to a GTA problem, assumption (A7) is thus a simplification. The effect of this simplification on the calculation results for GTA is still an open question.

\subsection{Cathode sheath (or space charge layer)}

This first sub-layer on the cathode surface is locally electrically charged. The charge fluxes taking place in the sheath, see Fig. 11, are the flux of electrons emitted from the cathode surface by thermionic or thermo-field emission $\varphi_{e m}$ and to a lesser extent by secondary emission $\varphi_{\text {sem }}$, and also the flux of ions $\varphi_{i}$ and back diffused electrons $\varphi_{b d}$. The cathode layer forms a potential barrier of voltage

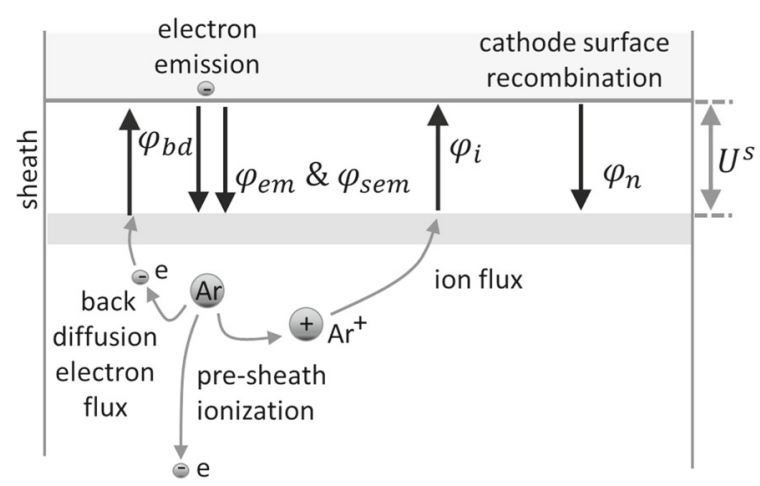

Fig. 11 Sketch of the particle fluxes in the cathode sheath: emitted electrons $\left(\varphi_{e m}\right)$, secondary emitted electrons $\left(\varphi_{\text {sem }}\right)$, ions $\left(\varphi_{i}\right)$, backdiffusion electrons $\left(\varphi_{b d}\right)$, and recombined atoms $\left(\varphi_{n}\right)$ 
drop $U^{s}$ that enhances electron emission, accelerates the emitted electrons and the incoming ions, and decelerates the back diffusing electrons. The number of collisions is small since $\lambda_{D}<\lambda_{c}$; this sub-layer is thus at the transition between being collisional and collisionless [49], and a continuum approach does not apply. The cathode sheath model combined with either a LTE-plasma in [25, 26], or a FNE-plasma in [11-14], is based on the following assumptions:

(A8) the cathode sheath is collisionless,

(A9) the emitted electrons are at thermal equilibrium with the cathode surface,

(A10) all the ions reaching the cathode surface are recombined,

(A11) the recombined ions are at thermal equilibrium with the cathode surface,

(A12) the space charge of emitted electrons is negligible when calculating the cathode surface electric field,

(A13) the temperature of the back diffusion electrons is constant across the sheath and is equal to the electron temperature at the sheath/pre-sheath interface,

(A14) the nearly isotropic distribution functions of the back diffusion electrons in the sheath can be approximated by a Maxwellian.

The validity of assumption (A8) was investigated by Benilov [49] and by Pekker and Hussary [19]. It can be seen in [19] that the ion collisional factor in the sheath decreases when the current density increases. For the current density range specific of GTA, this collisional factor is about 2 or less [19]. The cathode sheath of GTA is thus weakly collisional for ions. Benilov [49] did study the heating of refractory cathodes by high-pressure arc plasmas using both collisionless and collisional sheath models. It can be observed in [49] that at pressure close to the atmospheric pressure the results obtained assuming collisionless or collisional sheath show very little difference. Thus, although the cathode sheath of GTA is not strictly collisionless for ions, assumption (A8) can be made.

Assumption (A9) seems to be natural. Concerning assumption (A10), if a small fraction of ions colliding with the cathode surface does not recombine, it is not clear how to evaluate it. Assumption (A10) is thus accepted until further knowledge is acquired.

Concerning assumption (A11), the ions colliding with the cathode surface transfer part of their kinetic and thermal energy to the surface. If their residence time for recombination on the surface is sufficient they can fully thermalize with the surface. It might however happen that a small fraction of the recombined ions thermalize only partially with the cathode surface if the residence time is too short, but again it is not clear how to evaluate it.
The space charge of emitted electrons uses to be neglected when calculating the cathode surface electric field of GTA, e.g [2, 25]. This assumption was shown by Benilov [22] to be valid for high intensity discharge lamp, and recently questioned by Pekker and Hussary [19]. Figure 12 compares the sheath number density of ions, back-diffusion electrons, and thermionic and secondary emitted electrons calculated with the GTA test case of Appendix B. It can be seen that, at any location within the arc attachment area, the number of emitted electrons (thermionic and secondary) represents about $1 \%$ of the number of electrons in the sheath. It is thus justified to neglect the emitted electrons present in the sheath when calculating the cathode surface electric field. The generalization of this result to the overall cathode surface is straightforward. Assumption (A12) is thus satisfied.

Assumption (A13) is related to assumption (A8): an electrically charged and collisionless cathode sheath has an effect on the drift velocity of charged particles (due to the acceleration by the sheath drop voltage $U^{s}$ ) and no effect on the thermal velocity (due to the quasi absence of collision). Assumption (A14) is thus satisfied as a consequence of assumption (A5).

The cathode layer model consists in solving locally the energy conservation and the current density continuity at the cathode surface in order to determine the local cathode surface temperature, $T^{c / s}$, and the local cathode sheath voltage drop $U^{s}$ (see Fig. 10). For the GTA test case investigated in this study, the calculation results obtained on the arc axis lead to the cathode sheath voltage drop $U^{s}=5.4 \mathrm{~V}$. Also, the ionization layer (or pre-sheath) voltage drop is $U^{i}=0.3 \mathrm{~V}$, and the total voltage (defined as the sum of the

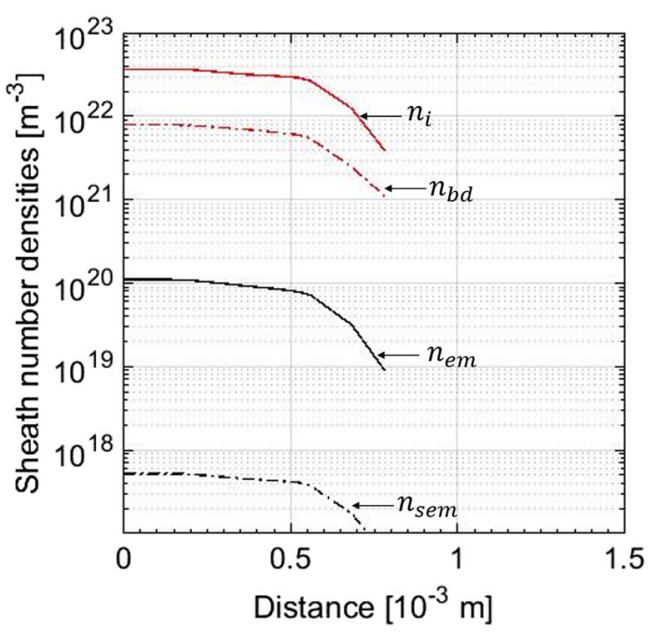

Fig. 12 Number density of ions $\left(n_{i}\right)$, back-diffusion electrons $\left(n_{b d}\right)$, thermionic emitted electrons $\left(n_{e m}\right)$, and secondary emitted electrons calculated in the sheath as functions of the distance from the cathode tip center 
cathode body, the cathode sheath and pre-sheath, and the arc column voltage) is $13.4 \mathrm{~V}$. This calculated arc voltage (that does not account for the anode sheath voltage) is close to the experimental measurement of 12.5-13 V reported by Haidar in [50]. The different heat fluxes are illustrated in Fig. 13. The arc attachment area corresponds to distances ranging from 0 to about $0.8 \times 10^{-3} \mathrm{~m}$. It can be seen in Fig. 13 (right) that, in the central part of the arc attachment area, radiative emission and absorption are both negligible compared to the heat fluxes transferred by ions, and by emitted and back diffusion electrons.

\subsection{Knudsen layer}

This layer accelerates the ions up to the Bohm velocity, which is the critical threshold to form the negatively charged cathode sheath [23]. It is kinetic and collisional. It should thus be modeled with Boltzmann-Fokker-Planck equations (with collisional operators), as done by Schmitz and Riemann [51]. However the computational time would then be too excessive for GTA applications. In both the LTE-plasma column approach of $[25,26]$ and the FNE-plasma column approach of $[11,14]$ the Knudsen layer is modeled in a simplified way, as proposed by Benilov [22], assuming that

(A15) the component normal to the cathode wall of the ion distribution function can be approximated by an Heaviside distribution function defined based on Bohm velocity.

Nandelstädt et al. [52] showed that the results obtained with a detailed modelling of the Knudsen layer (as done by Schmitz and Riemann [51]) are close to the results obtained with Benilov's simplified model. It implies that assumption (A15) is justified.

\subsection{Ionization layer (as cathode pre-sheath, or part of a FNE-plasma)}

The third sub-layer from the cathode surface is the region where charged species are produced by ionization, sustaining the electrical discharge. Important characteristics of this layer are diffusion, thermal non-equilibrium, and ionization non-equilibrium which is mainly due to charge diffusion [48]. Its modelling is different if the plasma column is in LTE or in FNE. For a LTE-plasma column (as well as for a 2T-plasma) a specific model is required for the ionization layer [25, 26]. For a FNE-plasma the ionization layer is modelled as the plasma. In any case one more closure relation needs to be established to determine the electron temperature at the sheath boundary on the plasma side. In the LTE-plasma approach [25, 26], this closure relation is the energy conservation equation through the ionization layer; it involves the heat fluxes and the electric work sketched in Fig. 14. The heat transfers to/from the cathode pre-sheath include the heat gain through the thermionic or thermo-field electrons, to lesser extent the secondary emitted electrons, the heat loss with the ions and back diffusion electrons moving away towards the sheath, and the heat loss with the electrons moving towards the plasma column. Electric work is also gained by the charged particles as they move through the pre-sheath potential drop $U^{i}$. In the FNE-plasma approach [11-14], the closure relation is instead the electron energy conservation through the sheath; it involves the heat fluxes and the electric work sketched in Fig. 15.

The ionization layer model of $[25,26]$ is based on the following main assumptions:

(A16) real gas effect can be neglected,

(A17) radiative ionization (photoionization) is negligible,
Fig. 13 Heat fluxes at the cathode surface due to emitted electrons $\left(q_{e m}\right)$, secondary emitted electrons $\left(q_{\text {sem }}\right)$, ions $\left(q_{i}\right)$, back-diffusion electrons $\left(q_{b d}\right)$, recombined atoms $\left(q_{n}\right)$, conduction $\left(q_{\text {cond }}\right)$, radiative emission $\left(q_{\mathrm{rad}, \mathrm{em}}\right)$ and absorption $\left(q_{\text {rad, abs }}\right)$; Left: sketch; Right: calculation results as functions of the distance from the cathode tip center

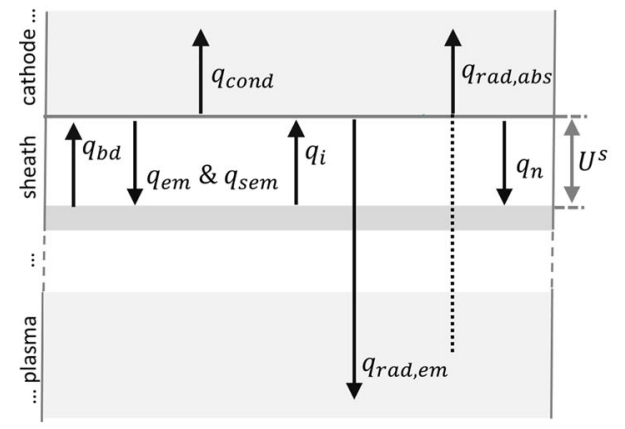




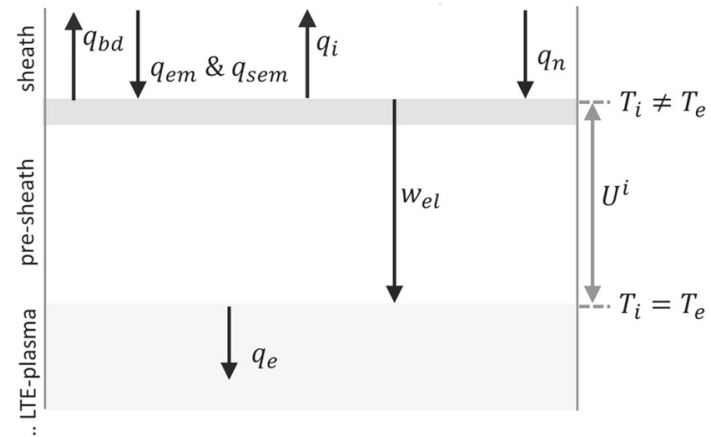

Fig. 14 Sketch of the heat fluxes through the pre-sheath boundaries due to emitted electrons $\left(q_{\mathrm{em}}\right)$, secondary emitted electrons $\left(q_{\mathrm{sem}}\right)$, ions $\left(q_{i}\right)$, back-diffusion electrons $\left(q_{b d}\right)$, recombined atoms $\left(q_{n}\right)$, electron flux towards the LTE-plasma $\left(q_{e}\right)$, and work done by the electric field $\left(w_{e l}\right)$

(A18) the ionization energy is provided by the impacting electrons,

(A19) the direct and reverse ionization reaction rates are assumed to obey a Saha law.

The assumptions (A16)-(A17) are justified since the pressure is of the order of the atmospheric pressure. Here, assumption (A16) simply implies that each plasma component (molecule, atom, ion) is thermally perfect and thus obeys the ideal equation of state. Assumptions (A16)-(A17) were also made in the FNE-plasma approach [2,11-14] (see [53]).

The reverse reaction to photoionization, namely radiative recombination, was included in the original FNEplasma model [53]. The recombination rate was calculated from recombination cross sections assuming local thermodynamic and Saha equilibrium; see Katsonis [54]. As any FNE-approach combines the ionization layer with the plasma column, it implies that radiative recombination was also modeled in the ionization layer. It was shown in [53] that below the cathode tip, at equidistance with the anode, the reaction rate of radiative recombination is one order of

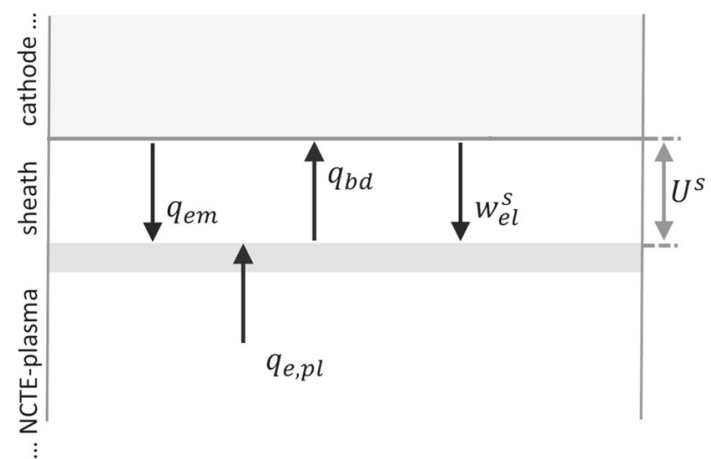

Fig. 15 Sketch of the electron heat fluxes through the sheath boundaries due to emitted electrons $\left(q_{e m}\right)$, back-diffusion electrons $\left(q_{b d}\right)$, electron flux from the FNE-plasma $\left(q_{e}, p l\right)$, and work done by the electric field $\left(w_{e l}^{s}\right)$ magnitude less than the reaction rate of impact ionization. It seems thus reasonable to extrapolate that in the ionization layer radiative recombination could be negligible. In the novel FNE-plasma model [2,11-14], the energy loss due to volumetric radiation was taken into account in the ionization layer. This was made including the free-free, free-bound and $4 \mathrm{p}-4 \mathrm{~s}$ line radiation, and expressing these radiation losses as a function of the electron and atom number densities according to Beulens et al. [55]. The GTA calculation results presented in $[2,14]$, show that the local radiation losses in the cathode vicinity lead to negligible heat fluxes compared to the other heat fluxes to the cathode. Radiative recombination was not taken into account in the ionization layer model (with LTE-plasma) of [25, 26]. Moreover, the energy loss due to volumetric radiation was only modeled in the LTE-plasma column, and not in the ionization layer.

For argon in a thermal plasma, and in regions with large enough electron number density, electron impact ionization, $A r+e^{-} \rightleftharpoons A r^{+}+2 e^{-}$, and impact ionization from excited states, $\mathrm{Ar}+e^{-} \rightleftharpoons A r^{\star}+e^{-} \rightleftharpoons A r^{+}+2 e^{-}$, are the main processes of ion production and recombination [56]. While the former reaction represents the main ionization channel at high electron temperature, the latter is important in the low electron temperature range $\left(T_{e}<10000 \mathrm{~K}\right)$ [57]. For GTA simulation purpose, these reactions need to be modelled at the macroscopic scale, based on the reaction rates. More than hundred years of experimental and theoretical studies were conducted for developing impact ionization reaction rates. Important advances were made studying thermal plasma in the field of re-entry flow for spacecraft and planetary probes, and to analyze astrophysical and laboratory plasmas; see $[58,59]$, and references therein. It did result in the development of several models of reaction rates, which were reviewed by Schmidt et al. [60] in the particular case of argon ionization. The ionization energy is mainly provided by the impacting electron if the electron number density is not too low. This condition is satisfied in the part of the ionization layer located in the arc attachment area. Assumption (A18) was made in both the LTE-plasma approach [25, 26] and the FNE-plasma approach [11-14], so that the reaction rates related to impact ionizations and their reverse recombination are solely function of the electron temperature. In $[25,26]$ impact ionization is modeled in the ionization layer using reaction rates calculated from partition functions. Ionization reactions resulting in multiplecharge ions $\left(\mathrm{Ar}^{2+}, \mathrm{Ar}^{3+}\right)$ are also included. The GTA calculation results show that in the ionization layer, the number density of $A r^{2+}$ is very small (less than $10^{18} \mathrm{~m}^{-3}$ ), and $A r^{3+}$ does not form [26]. Multiple-charge ions could thus be neglected in the ionization layer. The calculation results also show that at the ionization layer boundary on the sheath side (in the arc attachment region) the electron number density is of the order of $10^{23} \mathrm{~m}^{-3}$ [26], and the electron temperature 
$T_{e} \geq 10000 \mathrm{~K}$ (see Fig. 10). Impact ionization from excited states was not included in the ionization layer of this model.

In the FNE-plasma model (original [53], and novel [2, 11-14]), impact ionization from the four lowest 4s-excited states of argon atoms $A r^{\star}$ are also included. The resultant set of reactions was modeled using global reaction rates according to a concept developed by Hinnov and Hirschberg [61]. It assumes that above some threshold level (or bottleneck level) the electrons are in equilibrium with the free electrons, and the threshold can vary as a function of the electron temperature [2]. It should be noticed that the excitation of argon atoms in collisions with argon atoms, $A r+A r \rightleftharpoons A r^{\star}+A r$, was also included in [53], as well as a model extended to the $2 \mathrm{p} 10-2 \mathrm{p} 5,2 \mathrm{p} 4-2 \mathrm{p} 3,2 \mathrm{p} 2$ and $2 \mathrm{p} 1$ excited levels of $A r^{\star}$. The effect of the number of excited levels was investigated with the original FNE-approach and a free-burning argon arc at 200 A (cathode to anode distance of $8 \mathrm{~mm}$ ) [53]. The calculation results show that the extended model leads to a lower electron temperature (by about $2500 \mathrm{~K}$ ) at the cathode tip than the model restricted to the lowest $4 \mathrm{~s}$-excited states. Besides, the differences are observed to be negligible in the plasma core (in the thermal equilibrium region).

The assumption (A18) might be questioned at the transition region between electrically emitting and non-emitting cathode surface and in the non-emitting region where the electron density is expected to be low. At low electron density part of the ionization energy might indeed be provided by internal energy states of heavy particles that are not at equilibrium with the free electrons. To cover a range going from low to high electron number density, the reaction rates should depend on the so-called excitation temperature, or the effective temperature [62], which depends on both the electron and heavy particle temperature. But this raises a difficulty concerning the choice of appropriate expression for the excitation temperature, as further developed in [62]. To our knowledge, this problem is still open.

Assumption (A19) is most probably oversimplified. It is indeed known that impact ionization is not at equilibrium in the ionization layer. The ionization layer model based on assumptions (A16)-(A19) was developed by Cayla [27] and applied to an HID lamp studied experimentally by Dabringhausen et al. [63]. The calculated cathode fall voltage and the energy absorbed by the cathode were in good agreement with the experimental measurements. The calculated surface temperature was overestimating the experimentally measured temperature by about $250 \mathrm{~K}$; this result was considered to be within the limits of experimental error [27]. Comparisons with experimental measurements for a $100 \mathrm{~A}$ and $200 \mathrm{~A}$ argon GTA test case were made in [25]. When considering the inhomogeneous cathode surface physical state (assumption (A4b)) the calculation results show a good agreement with the cathode surface temperature measurements of Haidar and Farmer [30], and the plasma electron temperature measurements of Haddad and Farmer [29]. Nevertheless, it would be desirable to investigate further the validity of this assumption. A pre-sheath model taking into account ionization non-equilibrium was developed by Benilov [48] prior to the model based on the simplified assumptions (A19). To be easily applied to GTA (with non-uniform voltage drop along the cathode surface), this model would need to be reformulated in order to use the current density as input parameter. To our knowledge, this has not yet been done.

\section{Plasma column-modelling assumptions}

The thermal fluid model used for the LTE-plasma column $[25,26]$ assumes:

(A20) a Newtonian fluid,

(A21) a thermally expansible and mechanically incompressible fluid,

(A22) a laminar flow,

(A23) a net volumetric radiation density whose calculation can be decoupled from the calculation of the temperature field,

(A24) a one-fluid model in local chemical equilibrium, and

(A25) in local thermal equilibrium.

The assumptions (A20)-(A21) are known to be satisfied since the fluid is a plasma, and standard GTA operating conditions correspond to a small Mach number. Concerning the assumption (A22), GTA welding nozzles are designed to ensure a laminar flow regime in the interior of the torch. The standard process windows recommended for operation are also designed to maintain a laminar flow in the arc plasma jet. However, at the arc plasma edge, the mixing of the shielding gas with the surrounding atmosphere can be turbulent depending on, e.g., the value of the gas flow rate, the torch tilt, the welding speed. The effect of the gas flow rate was visualized by Schnick et al. [64] operating a $100 \mathrm{~A}$ GTA torch with Ar50/He50 shielding gas. At $10 \mathrm{l} / \mathrm{min}$, the mixing was observed to be laminar, while at $20 \mathrm{l} / \mathrm{min}$ the transition to turbulent mixing in the arc periphery was established. However, turbulent rather than laminar mixing in the arc fringes is not expected to result in a significant effect in the plasma core and in the cathode vicinity.

The assumption (A23) is weaker than the more common assumption of optically thin plasma. The radiation from a GTA is not optically thin at all wavelengths since some of the wavelengths are absorbed within the radiating volume. This self-absorption phenomenon was shown to be important for some shielding gases, e.g., Ar-He [65] and very 
important for metal vapor $[31,66]$. It can be accounted for in the net emission coefficients using appropriate methods decoupling the calculation of the net volumetric radiation density from the calculation of the temperature field. An example is the integral method of partial characteristics (IMPC) developed by Sevastyanenko and Bakken [67]. The method developed by Lowke et al. [68, 69] (with radiation from an homogeneous isothermal sphere of pre-defined effective radius) seems to be the most commonly used [62, $65,66]$. The FNE-plasma column model of $[11,14]$ is also based on the assumptions (A20)-(A23).

The simplifying assumptions (A24)-(A25) are made in the LTE-plasma model developed by Javidi Shirvan et al. $[25,26]$. These assumptions are known to be valid in the high temperature plasma core. They are inappropriate in the colder plasma regions where the temperature goes below some threshold (about $12500 \mathrm{~K}$ for an argon plasma [70]). It is well known that for GTA the colder plasma regions are in the cathode (and anode) vicinity and at the plasma fringes. Deviation from thermal equilibrium in the cathode vicinity is not neglected in $[25,26]$ since it is modeled in the ionization layer (see Section 3.3); however, it might extend beyound the ionization layer. Deviation from chemical equilibrium in the ionization layer was discussed above. Deviation from both thermal and chemical equilibrium is neglected in the plasma fringes. The simplifying assumptions (A24)-(A25) were not used in the FNE-plasma models developed by Baeva et al. [12, 14]. The comparisons made by Benilov et al. [11] showed that deviation from chemical equilibrium in the plasma fringes has a negligible effect on the plasma column temperature. It is assumed that similar conclusions should hold for deviation from chemical equilibrium in the plasma fringes. This point is completed later on in this section.

The electromagnetic part of the model is derived from the set of Maxwell equations supplemented with a generalized Ohm's law [71] doing the following assumptions:

(A26) the Debye length $\lambda_{D}$ is much smaller than the characteristic length of the welding arc so that local electro-neutrality is verified,

(A27) the characteristic time and length of the welding arc allow neglecting the displacement current compared to the current density,

(A28) the Larmor frequency is much smaller than the average collision frequency of electrons, implying that the Hall current is negligible,

(A29) the magnetic Reynolds number is much smaller than unity, implying that the induction current is negligible, and

(A30) the diffusion and thermodiffusion currents due to electrons are negligible compared with the drift current.

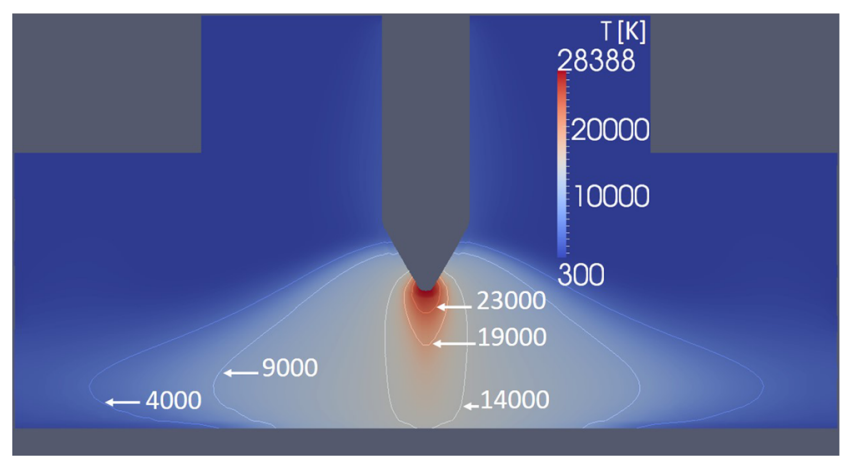

Fig. 16 Temperature field calculated in the plasma with assumption (A4b) and the LTE-approach

The assumptions (A26)-(A30) are known to be verified in the LTE-plasma column [71]. It should be noticed that assumption (A30) is not satisfied in the ionization layer, contrary to (A26)-(A29). Assumption (A30) is not used in the novel FNE-plasma model developed by Baeva et al. [14]. Diffusion and thermodiffusion currents are indeed important to perform a consistent energy and current density coupling between plasma column and cathode layer when the ionization layer is modeled as part of the plasma column.

The temperature calculated in the plasma column using the LTE-plasma approach with assumption (A4b) is plotted in Fig. 16 (test case of Appendix B). The plasma temperature calculated with the assumption (A4a) and (A4b), corresponding respectively to the homogeneous and nonhomogeneous cathode surface, are plotted in Figs. 17 and 18. The experimental measurements of the electron temperature made by Haddad and Farmer [29] are also reported in these figures. In Fig. 17, the plot is done along the arc axis, and in Fig. 18 it is along the radial direction at various distances below the cathode tip. It can be seen that the local physical state of the thoriated tungsten cathode has a significant effect on the calculated plasma temperature field. The calculation results obtained accounting for the non-homogeneity of this physical state agree better with

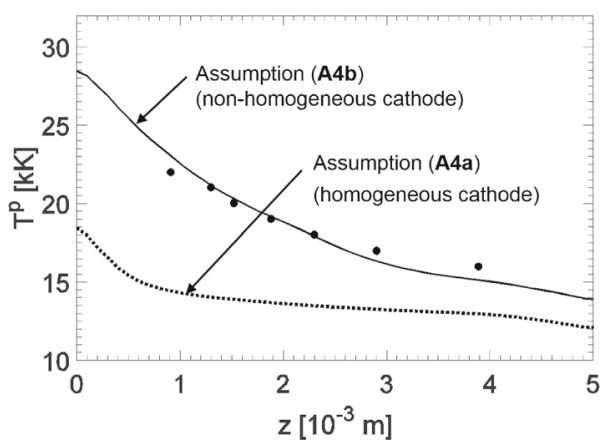

Fig. 17 Temperature along the arc axis as a function of the distance from the tip center: electron temperature measured by Haddad and Farmer [29] (•), and calculated LTE-plasma temperature 
the experimental measurements. In other words, the nonuniformity of the surface distribution in rare earth electron emitters, which is induced by the arc-cathode interaction, is important to consider (i.e., assumption (A4b)).

It is however observed in Fig. 18 (top and center) that the calculated temperature deviates from the experimental measurements when the plasma temperature is less than about $12500 \mathrm{~K}$. Below this temperature deviation from local thermal equilibrium becomes important, as underlined by, e.g., Haidar [30]. Extending the plasma model from local thermal equilibrium (LTE) to two-temperatures (2T) would enlarge the model validity to a wider part of the plasma column, and to the upper part of the cathode surroundings (in the cathode region too cold to emit electrons). In this
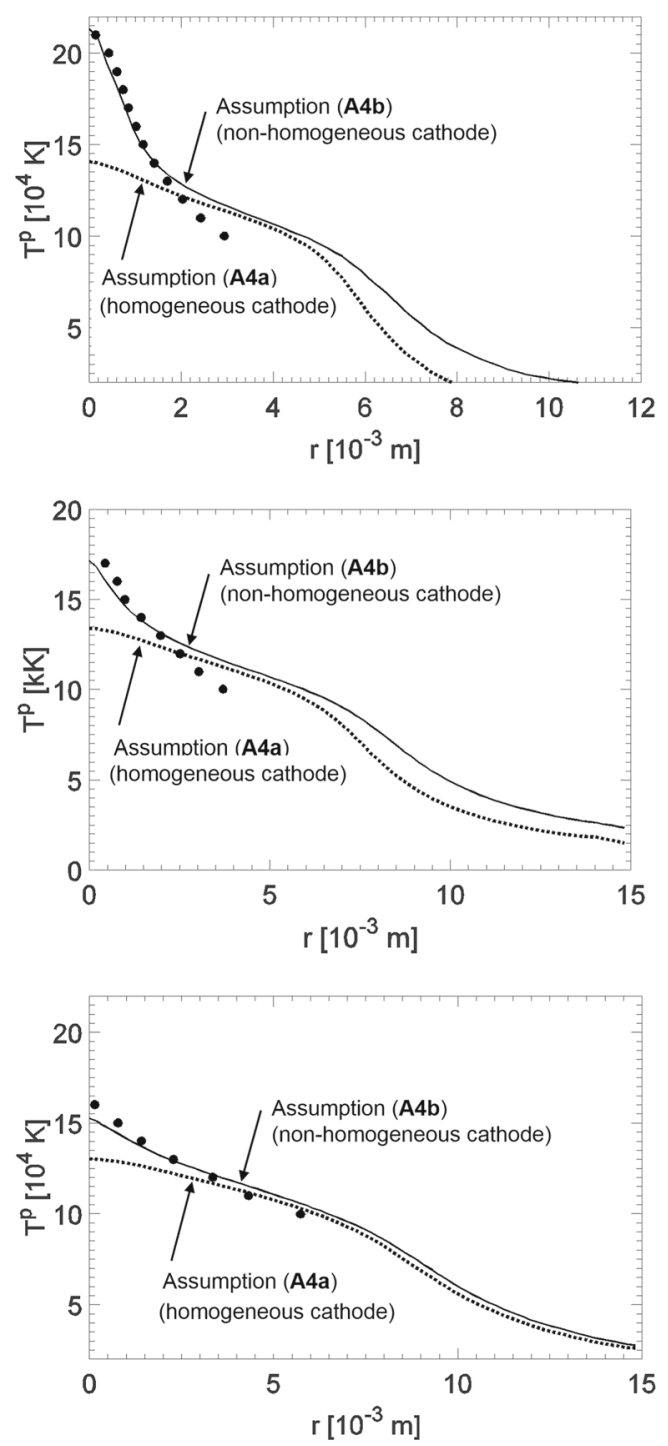

Fig. 18 Temperature along the radial direction: electron temperature measured by Haddad and Farmer [29] (•), and calculated LTE-plasma temperature. Top: at $z=1.25 \mathrm{~mm}$, Center: at $z=2.5 \mathrm{~mm}$, and Bottom: at $z=3.75 \mathrm{~mm}$ from the tip center last region, the LTE-approach is too approximate to allow coupling the plasma column to the ionization layer model. A 2T-approach would be more appropriate. However, a 2Tapproach is not expected to provide as accurate results as a FNE-approach in the arc fringes. On the other hand, the arc fringes should contribute little to the arc properties, and a 2T-approach might have the advantage of a lower computational time than a FNE-approach since it involves a lower number of conservation equations to be solved in the plasma domain. This still need to be investigated quantitatively.

One important aspect when extending a LTE approach to a two-temperature approach (either $2 \mathrm{~T}$ or FNE) is the way the conservation equation for the total energy is distributed into an energy conservation equation for electrons and one for heavy particles. Various distributions were developed and are still being used. Freton et al. [72] studied several of them. These authors focused on distributions such that the conservation equation for the total energy has the same expression in both the LTE and the $2 \mathrm{~T}$ approach (this basic requirement is not satisfied by all the developed models). They did show that these different distributions are not equivalent and can lead to non-physical results. Freton et al. also derived a new one from a system of Botzmann equations [72]. These authors analyzed systematically this problem and found possible causes related to the distribution of thermal conductivity and ionization energy. The reactive thermal conductivity due to ionization reactions should be assigned to the electron thermal conductivity [72, 73]. The ionization energy should be accounted for in the electron rather than the heavy particle energy conservation [72]. It can be seen in [14] that the FNE model satisfies this condition.

\section{Conclusion}

Several recent developments in the field of gas tungsten arc modeling including the physics of the cathode layer were summarized and discussed. Hopefully most of the modelling assumptions were examined. Part of the modelling assumptions could be justified, while the remaining part is believed to be oversimplified. Part of the oversimplified assumptions are believed to have little effect on the main arc properties. Among the oversimplified assumptions that could have an effect on arc properties of importance for welding applications, e.g., the heat fluxes to the workpiece, main issues that remain unexplored concern

- The effect of plasma radiation absorbed by the cathode surface when accounting for the cathode layer all over the cathode surface, i.e., over both the electrically conducting and the electrically non-conducting part of the cathode surface. 
- Within the frame of a LTE or 2T-plasma approach, the effect of the non-uniformity of the ionization layer thickness.

- Within the frame of a LTE or 2T-plasma approach, the reformulation of the ionization layer model developed by Benilov [48] in order to use the current density as input parameter; the study of its effect on GTA compared to the simpler (in terms of physical assumptions) ionization layer model developed by Cayla [27].

- The effect of different models rather recently developed [60] for the reaction rates of impact ionization, including ionization from excited states, and their reverse recombination reactions.

- The effect of activator vaporization, first on the cathode layer and the plasma column, and next on the formation of new physical states on the cathode surface (see e.g. $[25,35])$.

- The understanding of the diffusion mechanisms of rare earth metal oxides other than thorium.

Acknowledgments This work was supported by KK-foundation in collaboration with ESAB. These supports are gratefully acknowledged. The author is grateful to Dr. Margarita Baeva and to the Journal of Plasma Chemistry and Plasma Processing for permission to reproduce figures from [2].

Open Access This article is distributed under the terms of the Creative Commons Attribution 4.0 International License (http://creative commons.org/licenses/by/4.0/), which permits unrestricted use, distribution, and reproduction in any medium, provided you give appropriate credit to the original author(s) and the source, provide a link to the Creative Commons license, and indicate if changes were made.

\section{Appendix A: Model}

The thermo-fluid model in the LTE-plasma column is given by

$$
\begin{aligned}
& \quad \partial_{t} \rho+\nabla \cdot(\rho \vec{u})=0, \\
& \partial_{t}(\rho \vec{u})+\nabla \cdot(\rho \vec{u} \otimes \vec{u}) \\
& =\nabla \cdot\left[\mu\left(\nabla \vec{u}+(\nabla \vec{u})^{T}\right)-\frac{2}{3} \mu(\nabla \cdot \vec{u}) \mathbf{I}\right]-\nabla P+\vec{j} \times \vec{B}, \\
& \partial_{t}(\rho h)+\nabla \cdot(\rho \vec{u} h)=\nabla \cdot\left(\frac{\kappa^{p}}{C_{p}} \nabla h\right) \\
& \quad+\vec{u} \cdot \nabla P+\vec{j} \cdot \vec{E}-S_{\text {rad }}+\nabla \cdot\left[\frac{5 k_{b} \vec{j} h}{2 e C_{p}}\right],
\end{aligned}
$$

where $\rho=\rho\left(T^{p}\right)$ denotes the plasma density, $\vec{u}$ the velocity, $P$ the pressure, $h$ the specific enthalpy, $T^{p}$ the temperature, $\mu=\mu\left(T^{p}\right)$ the viscosity, $\mathbf{I}$ the identity tensor, and $\vec{B}$ the magnetic field. $\kappa^{p}=\kappa^{p}\left(T^{p}\right)$ is the thermal conductivity, $S_{\text {rad }}=S_{\text {rad }}\left(T^{p}\right)$ the radiation heat loss [74], and $C_{p}=C_{p}\left(T^{p}\right)$ the specific heat at constant pressure. The thermodynamic and transport properties are linearly interpolated from tabulated data derived by Rat et al. [75].

The electromagnetic model is given by

$\nabla \cdot(\sigma \nabla V)=0$

$\triangle \vec{A}=\mu_{o} \sigma \nabla V$

where $V$ is the electric potential, $\sigma$ the electric conductivity, $\vec{A}$ the magnetic potential, and $\mu_{o}$ the permeability of free space. $\sigma$ is set to $\sigma^{p}\left(T^{p}\right)$ in the thermal plasma. The current density $\vec{j}$, the magnetic field $\vec{B}$ and the electric field $\vec{E}$ are derived according to

$\vec{B}=\nabla \times \vec{A}, \quad \vec{E}=-\nabla V, \quad$ and $\quad \vec{j}=\sigma \vec{E}$

The cathode body temperature $T^{c}$ is governed by

$\partial_{t}\left(\rho^{c} C_{p} T^{c}\right)=\nabla \cdot\left(\kappa^{c} \nabla T^{c}\right)+\vec{j} \cdot \vec{E}$

where $\rho^{c}$ denotes the density, $C_{p}=C_{p}\left(T^{c}\right)$ the specific heat, and $\kappa^{c}=\kappa^{c}\left(T^{c}\right)$ the thermal conductivity of the cathode material. The current density $\vec{j}$ and the electric field $\vec{E}$ in the cathode are given by Eqs. 4 and 6, replacing the electric conductivity $\sigma$ with the cathode electric conductivity $\sigma^{c}\left(T^{c}\right)$. Equation 5 is also solved in the cathode core to set the boundary conditions for $\vec{A}$ in the plasma at the cathode surface.

The cathode layer model is made of two sets of equations. The first set provides the plasma composition in the ionization layer. It is made of three Saha equations $(l=1,2,3)$ [76], supplemented with Dalton's law and local charge neutrality

$$
\begin{aligned}
\frac{n_{e}^{p s / s} n_{A r^{l+}}^{p s / s}}{n_{A r^{p s-1)+}}^{p s / s}}= & 2 \frac{Q_{A r^{l+}}\left(T_{e}^{p s / s}\right)}{Q_{A r^{(l-1)+}}\left(T_{e}^{p s / s}\right)} \frac{\left(2 \pi m_{e} k_{b} T_{e}^{p s / s}\right)^{\frac{3}{2}}}{h_{p}^{3}} \\
& \times \exp \left(-\frac{E_{i, A r^{(l-1)+}-\Delta E_{l}}}{k_{b} T_{e}^{p s / s}}\right),
\end{aligned}
$$

$P=\sum_{l=0}^{3} n_{A r}^{p s / s} k_{b} T_{h}^{p s / s}+n_{e}^{p s / s} k_{b} T_{e}^{p s / s}$

$n_{A r}^{p s / s}=\sum_{l=1}^{3} n_{A r^{l+}}^{p s / s}$.

$n_{A r^{l+}}^{p s / s}$ is the the number density of the ion $\mathrm{Ar}^{l+}$ at the presheath boundary on the sheath edge. $n_{e}^{p s / s}=\sum_{l=1}^{3} l n_{A r^{l+}}^{p s / s}$ and $n_{i}^{p s / s}=\sum_{l=1}^{3} n_{A r}^{p s / s}$ are respectively the electron number density and total ion number density at the pre-sheath boundary on the sheath side. $Q_{A r^{l+}}$ denotes the internal 
partition function of the heavy particle $\mathrm{Ar}^{l+}$. The online toolbox of the National Institute of Standard and Technology [77] is employed to retrieve the data needed to tabulate the internal partition function for argon atom and ions. $e$ is the electron elementary charge, $m_{e}$ the electron mass, $k_{B}$ the Boltzmann constant, and $h_{p}$ the Planck constant. $\bar{E}_{i}=\sum_{l=1}^{3} n_{A r l+}^{p s / s} E_{i, A r^{l+}}$ is the average ionization energy, where $E_{i, A r^{l+}}$ denotes the ionization energy of $A r^{(l-1)+}+$ $e \rightarrow A r^{l+}+2 e . \Delta E_{l}=l e^{2} /\left(4 \pi \epsilon_{o} \lambda_{D}\right)$ is the lowering of the ionization energy, where $l=1,2,3$ and $\epsilon_{o}$ is the permittivity of free space $[78,79]$. The electron and ion number densities at the pre-sheath boundary on the plasma side are obtained in a similar way replacing in Eqs. 8-10 the temperatures by the plasma temperature.

The second set of equations includes the current continuity at the cathode surface, the energy balance at the cathode surface, and the energy conservation in the cathode pre-sheath. It reads

$j^{c / s}=e\left(Z \varphi_{i}+\varphi_{e m}+\varphi_{s e m}-\varphi_{b d}\right)$,

$q_{\text {cond }}=q_{i}+q_{b d}+q_{\text {rad }}^{a b s}-q_{e m}-q_{s e m}-q_{\text {rad }}^{e m}-q_{n}$,

$$
\begin{aligned}
\left(\varphi_{e m}+\varphi_{s e m}\right) & {\left[2 k T^{c / s}+e U^{s}+k T_{e}^{p s / s}\left(\ln \left(\frac{n_{e}^{p s / p}}{n_{e}^{p s / s}}\right)-3.2\right)\right] } \\
= & \varphi_{b d}\left[e U^{s}+k T_{e}^{p s / s}\left(\ln \left(\frac{n_{e}^{p s / p}}{n_{e}^{p s / s}}\right)-1.2\right)\right] \\
& +\varphi_{i}\left[2 k T_{i}^{p s / s}+\bar{E}_{i}+Z k T_{e}^{p s / s}\left(3.7-0.5 \ln \left(\frac{n_{e}^{p s / p}}{n_{e}^{p s / s}}\right)\right)\right],
\end{aligned}
$$

$q_{\text {cond }}$ denotes the conduction heat flux in the cathode, $Z=$ $n_{e}^{p s / s} / n_{i}^{p s / s}$ is the average ion charge. The electron emission flux from the cathode surface is given by the RichardsonDushman law

$\varphi_{\text {em }}=\frac{\lambda_{R} A_{0}\left(T^{c / s}\right)^{2}}{e} \exp \left(-\frac{\phi_{e f f}}{k_{b} T^{c / s}}\right)$,

where $T^{c / s}$ is the cathode surface temperature. $\phi_{\text {eff }}=e \phi-$ $\Delta \phi$ is the effective work function, $\phi$ the work function (in $\mathrm{eV}$ ), and $\Delta \phi$ the Schottky correction (in J). $\lambda_{R}$ is a material specific factor (see Table 1), and $A_{0}=4 \pi m_{e} k_{b}^{2} e / h_{p}^{3}$ the Richardson constant. The Schottky correction is written as $\Delta \phi=\sqrt{\left(e^{3} E^{c / s}\right) /\left(4 \pi \epsilon_{o}\right)}$. The electric field at the cathode surface [21] is given by

$E^{c / s}=\sqrt{\frac{2 n_{i}^{p s / s}}{\epsilon_{o}}\left[m_{i}\left(\frac{v_{+}^{3}-v_{-}^{3}}{6 v_{i}}-v_{B}^{2}-\frac{1}{3} v_{i}^{2}\right)-Z k_{b} T_{e}^{p s / s}\left(1-\exp \left(-\frac{e U^{s}}{k_{b} T_{e}^{p s / s}}\right)\right)\right]}$

where $v_{ \pm}=\sqrt{\left(v_{B} \pm v_{i}\right)^{2}+2 e Z U^{s} / m_{i}}$, and $m_{i}$ is the ion mass. The ion thermal velocity is $v_{i}=\sqrt{k_{b} T_{i}^{p s / s} / m_{i}}$, and the Bohm velocity $v_{B}=\sqrt{k_{b}\left(T_{i}^{p s / s}+Z T_{e}^{p s / s}\right) / m_{i}}$ [22]. The ion flux reads [22]

$\varphi_{i}=n_{i}^{p s / s} v_{B}$.

The flux of back-diffusion electrons is [22]

$\varphi_{b d}=\frac{n_{e}^{p s / s} v_{e}}{\sqrt{2 \pi}} \exp \left(-\frac{e U^{s}}{k_{b} T_{e}^{p s / s}}\right)$,

with the electron thermal velocity $v_{e}=\sqrt{k_{b} T_{e}^{p s / s} / m_{e}}$.

The radiation heat flux absorbed by the cathode surface is $q_{r a d}^{a b s}=\alpha q_{r a d}^{i n c}$ where the absorptivity $\alpha$ is set to 0.5 for incandescent tungsten $[33,34]$. The incident plasma radiation heat flux reaching the cathode surface is approximated using the view factor method [80-82],

$q_{\text {rad }}^{i n c}=\int_{\mathcal{V}} \frac{S_{\text {rad }}}{4 \pi r_{i j}^{2}} \cos \psi d \mathcal{V}_{j}$,

where $r_{i j}$ represents the distance between the differential area $i$ of the cathode surface and each differential volume $j$ of the plasma, and $\psi$ the view factor angle between the elements $i$ and $j$. The radiation heat flux emitted locally by the cathode surface is expressed using the gray body model

$q_{\text {rad }}^{e m}=q_{r a d}^{e m}\left(T^{c / s}\right)=\epsilon \sigma_{s}\left(T^{c / s}\right)^{4}$,

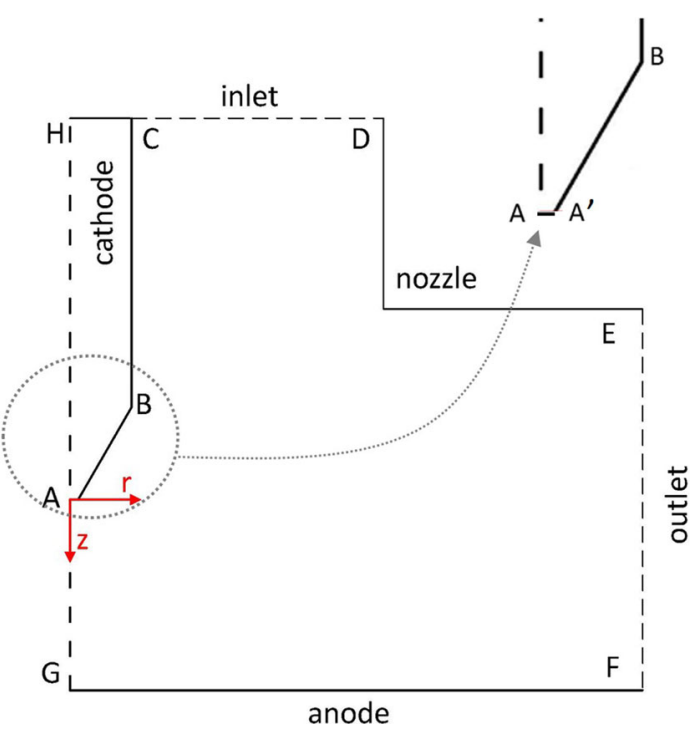

Fig. 19 Schematic sketch of the computational domain, and cathode tip detail (top right) 
Table 2 Boundary conditions for the cathode region. $n$ is the local unit vector normal to the boundary and outwards. $\mathbf{A}^{c}, \mathbf{A}^{p}$ are at the cell centers of the cathode and plasma boundary cells, respectively

\begin{tabular}{llll}
\hline & $\mathrm{AB}$ & $\mathrm{BC}$ & $\mathrm{CH}$ \\
\hline$T$ & $\partial_{n} T^{c}=q^{c l \rightarrow c} / \kappa^{c}\left(T^{c}\right)$ & $\partial_{n} T^{c}=0$ & $T_{i n}^{c}$ \\
$V$ & $\partial_{n} V=-j^{c / s} / \sigma^{c}\left(T^{c}\right)$ & $\partial_{n} V=0$ & $\partial_{n} V=-j_{i n}^{c} / \sigma^{c}\left(T_{i n}^{c}\right)$ \\
$\mathbf{A}$ & $0.5\left(\mathbf{A}^{c}+\mathbf{A}^{p}\right)$ & $0.5\left(\mathbf{A}^{c}+\mathbf{A}^{p}\right)$ & $\partial_{n} \mathbf{A}=0$ \\
\hline
\end{tabular}

where $\sigma_{s}$ is the Stefan-Boltzmann constant, and $\epsilon$ the cathode material emissivity. For tungsten the temperature dependent emissivity can be written as $\epsilon=\epsilon\left(T^{c / s}\right)=$ $-0.0266+1.8197 \times 10^{-4} T^{c / s}-2.1946 \times 10^{-8}\left(T^{c / s}\right)^{2}$, where the local cathode surface temperature $T^{c / s}$ is in Kelvin [83]. This expression is known to be in good agreement with experimental measurements for a temperature range from 400 to $3600 \mathrm{~K}$ [83].

The ion and the electron back diffusion heat fluxes towards the cathode surface are respectively [22]

$q_{i}=\varphi_{i}\left(2 k_{b} T_{i}^{p s / s}+\frac{1}{2} Z k_{b} T_{e}^{p s / s}+Z e U^{s}+\bar{E}_{i}-Z \phi_{e f f}\right)$

and

$q_{b d}=\varphi_{b d}\left(\phi_{e f f}+2 k_{b} T_{e}^{p s / s}\right)$.

The electron emission heat flux from the cathode surface is given by [22]

$q_{\text {em }}=\varphi_{\text {em }}\left(\phi_{\text {eff }}+2 k_{b} T^{c / s}\right)$.

The model is further detailed in $[25,26]$. The numerical procedure is described in [26]. The model was implemented in the open source computational fluid dynamic software OpenFOAM $^{\circledR}$.

\section{Appendix B: Test case}

The 200 A GTA test case was set to compare the calculation results to the experimental measurements of $[29,30]$. The shielding gas is argon at $P_{\text {atm }}=1.01 \times 10^{5} \mathrm{~Pa}$, and the cathode material is $\mathrm{W}-2 \% \mathrm{wt} \mathrm{ThO}_{2}$. The water cooled copper anode is reduced to a boundary condition. A schematic view of the computational domain is illustrated in Fig. 19. The domain dimensions are $(\mathrm{HG})=(\mathrm{GF})=15 \mathrm{~mm},(\mathrm{AG})=$ $5 \mathrm{~mm},(\mathrm{AH})=10 \mathrm{~mm},(\mathrm{HC})=3.2 \mathrm{~mm},(\mathrm{HD})=5 \mathrm{~mm}$. The nozzle wall (DE) has a vertical section of $5 \mathrm{~mm}$ and an horizontal section of $6.8 \mathrm{~mm}$. The conical tip has an angle of $60^{\circ}$; it is truncated at the tip radius $0.2 \mathrm{~mm}$ (at A). The tip edges located in A' and B are smooth with a radii of 0.174 $\mathrm{mm}$ and $0.746 \mathrm{~mm}$, respectively.

The computational domain is discretized with an unstructured mesh, except at the cathode-plasma interface where a structured mesh with hexahedral cells is used. The first row of cells on the cathode surface (in the plasma domain) has a uniform size along the direction normal to the cathode surface (A7). This size is set to $200 \mu \mathrm{m}$ to contain the cathode layer whose thickness is evaluated using the ionization length at the cathode tip end [48].

The boundary conditions applied in the cathode and the plasma arc regions are given in Tables 2 and 3. The argon gas enters the plasma domain with the volume flow rate $\dot{V}_{i n}^{p}=10 \mathrm{~L} / \mathrm{min}$, and the inlet temperature $T_{i n}^{p}=300 \mathrm{~K}$, through the nozzle inlet (CD) of total cross-sectional area $A_{i n}^{p}$. The electrons enter the top cross-sectional area $A_{i n}^{c}$ of the cathode, which is at temperature $T_{i n}^{c}=1000 \mathrm{~K}$, with the uniform current density $j_{i n}^{c}=I / A_{i n}^{c}$.

The boundary conditions for $\mathrm{V}$ and $\mathrm{T}$ at the cathode tip (AB) are divided into two parts, a part with arc attachment and a part without arc attachment. The boundary conditions in Tables 2 and 3 represent the part with arc attachment. For the part without arc attachment a zero gradient electric potential is set for both the cathode and the plasma region. Further details about the coupling boundary conditions can be found in [26].

\section{References}

1. Murphy AB (2015) A perspective on arc welding research: The importance of the arc, unresolved questions and future directions. Plasma Chem Plasma Process 35:471-489

2. Baeva M (2017) Non-equilibrium modeling of Tungsten-Inert Gas arcs. Plasma Chem Plasma Process 37:341-370

3. Murphy AB, Tanaka M, Tashiro S, Sato T, Lowke JJ (2009) A computational investigation of the effectiveness of different

Table 3 Boundary conditions for the plasma region. $n$ is the local unit vector normal to the boundary and outwards. $\mathbf{A}^{c}, \mathbf{A}^{p}$ are at the cell centers of the cathode and plasma boundary cells, respectively

\begin{tabular}{lllllll}
\hline & $\mathrm{AB}$ & $\mathrm{BC}$ & $\mathrm{CD}$ & $\mathrm{DE}$ & $\mathrm{EF}$ & $\mathrm{FG}$ \\
\hline $\mathbf{u}$ & $\mathbf{0}$ & $\mathbf{0}$ & $-\dot{V}_{i n}^{p} / A_{i n}^{p} \mathbf{n}$ & $\mathbf{0}$ & $\partial_{n} \mathbf{u}=\mathbf{0}$ & $\mathbf{0}$ \\
$P$ & $\partial_{n} P=0$ & $\partial_{n} P=0$ & $\partial_{n} P=0$ & $\partial_{n} P=0$ & $P_{\text {atm }}$ & $\partial_{n} P=0$ \\
$T$ & $T^{p / c l}$ & $\partial_{n} T^{p}=0$ & $T_{i n, p}$ & $\partial_{n} T^{p}=0$ & $300 \mathrm{~K}$ & $1000 \mathrm{~K}$ \\
$V$ & $\partial_{n} V=j^{c / s} / \sigma^{p}\left(T_{e}^{p s / s}\right)$ & $\partial_{n} V=0$ & $\partial_{n} V=0$ & $\partial_{n} V=0$ & $\partial_{n} V=0$ & 0 \\
$\mathbf{A}$ & $0.5\left(\mathbf{A}^{c}+\mathbf{A}^{p}\right)$ & $0.5\left(\mathbf{A}^{c}+\mathbf{A}^{p}\right)$ & $\partial_{n} \mathbf{A}=\mathbf{0}$ & $\partial_{n} \mathbf{A}=\mathbf{0}$ & $\mathbf{0}$ & $\partial_{n} \mathbf{A}=\mathbf{0}$ \\
\hline
\end{tabular}


shielding gas mixtures for arc welding. J Phys D Appl Phys 42(115205): 14

4. Lago F, Gonzalez JJ, Freton P, Gleizes A (2002) A numerical modelling of an electric arc and its interaction with the anode: Part I. The two-dimensional model. J Phys D Appl Phys 37:883-897

5. Heberlein J, Mentel J, Pfender E (2010) The anode region of electric arcs: a survey. J Phys D Appl Phys 43(2):023001 (31pp)

6. Sanders NA, Pfender E (1984) Measurement of anode falls and anode heat transfer in atmospheric pressure high intensity arcs. J Appl Phys 55:714-722

7. Luhmann J, Lichtenberg S, Langenscheidt O, Benilov MS, Mentel J (2002) Determination of HID electrode falls in a model lamp: II Langmuir-probe measurements. J Phys D Appl Phys 35:16311638

8. Nemchinsky VA, Peretts LN (1977) Anode sheath in a highpressure, high-current arc. Soviet Phys Tech Phys 22:1083-1087

9. Chazelas C, Trelles J-P, Choquet I, Vardelle A (2017) Main issues for a fully predictive plasma spray torch model and numerical considerations. Plasma Sources Sci Technol 37:627-651

10. Javidi Shirvan A, Choquet I (2016) GTAW Process - a review of cathode plasma coupling modelling. Welding in the World 60:821-835

11. Benilov MS, Almeida NA, Baeva M, Cunha MD, Benilova LG, Uhrlandt D (2016) Account of near-cathode sheath in numerical models of high-pressure arc discharges. J Phys D Appl Phys 49:215201(12pp)

12. Baeva M, Kozakov R, Gorchakov S, Uhrlandt D (2012) Twotemperature chemically non-equilibrium modelling of transferred arcs. Plasma Sources Sci Technol 21:055027(13pp)

13. Uhrlandt D, Baeva M, Pipa A, Kozakov R (2015) Cathode fall voltage of TIG arcs from a non-equilibrium arc model. Welding in the World 59:127-135

14. Baeva M, Benilov MS, Almeida NA, Uhrlandt D (2016) Novel non-equilibrium modelling of a DC electric arc in argon. J Phys D Appl Phys 49:245205(16pp)

15. Baeva M, Uhrlandt D, Benilov MS, Cunha MD (2013) Comparing two non-equilibrium approaches to modelling of a free-burning arc. Plasma Sources Sci Technol 22:065017(9pp)

16. Almeida NA, Benilov MS, Naidis GV (2008) Unified modelling of near-cathode plasma layers in high-pressure arc discharges. $\mathbf{J}$ Phys D Appl Phys 41:245201(26pp)

17. Baeva M, Uhrlandt D (2016) Analysis of the power budget of TIG arc based on non-equilibrium model. Paper given at study group 212 of the 2016 annual congress of the international institute of welding, 11-13 July 2016, Melbourn, Australia

18. Nemchinsky V (2014) Erosion of thermionic cathodes in welding and plasma arc cutting systems. IEEE Trans Plasma Sci 42:199-215

19. Pekker L, Hussary N (2015) Boundary conditions at the walls with thermionic electron emission in two temperature modeling of "thermal" plasmas. Phys. Plasma 22:083510(12 pp)

20. Pekker L, Murphy AB (2016) Boundary conditions at the ablative walls in two-temperature modelling of thermal plasmas with reactive working gas. J Phys D Appl Phys 49:375202(14pp)

21. Benilov MS, Marotta A (1995) A model of the cathode region of atmospheric-pressure arcs. J Phys D Appl Phys 28:1869-1882

22. Benilov MS (2008) Understanding and modelling plasmaelectrode interaction in high-pressure arc discharges: a review. J Phys D Appl Phys 41:144001(30pp)

23. Riemann K-U (1991) The Bohm criterion and sheath formation. J Phys D Appl Phys 24:493-518

24. Godyak VA, Sternberg N (1990) Smooth plasma-sheath transition in a hydrodynamic model. IEEE Trans Plasma Sci 18:159-168

25. Javidi Shirvan A, Choquet I, Nilsson H (2016) Effect of cathode model on arc attachment for short high-intensity arc on a refractory cathode. J Phys D Appl Phys 49:485201(17pp)
26. Javidi Shirvan A (2016) Modelling of cathode-plasma interaction in short high-intensity electric arc. Application to Gas Tungsten Arc welding. PhD. Thesis Chalmers University of Technology, Gothenburg

27. Cayla F (2008) Modélisation de l'interaction entre un arc électrique et une cathode. PhD Thesis Paul Sabatier University, Toulouse

28. Cayla F, Gonzalez J-J, Freton P, Teulet P (2008) Arc/cathode interaction model. In: Proceedings of 17th IEEE international conference on gas discharges and their applications, pp 157160

29. Haddad GN, Farmer AJD (1985) Temperature measurements in gas tungsten arcs. Weld J 64:339s-342s

30. Haidar J, Farmer AJ (1995) Surface temperature measurements for tungsten-based cathodes of high-current free-burning arcs. J Phys D Appl Phys 28:2089-2094

31. Murphy AB (2010) The effects of metal vapour in arc welding. J Phys D Appl Phys 43:434001(31pp)

32. Benilov MS, Cunha MD (2013) Joule heat generation in thermionic cathodes of high-pressure arc discharges. J Phys D Appl Phys 113:063301(11pp)

33. Larrabee R (1957) The spectral emissivity and optical properties of tungsten. Technical report 328, Research Laboratory of Electronics Massachusetts Institute of Technology, Cambridge

34. Nomerovannaya LV, Kirillova MM, Noskov MM (1971) Optical properties of tungsten monocrystals. Soviet Phys JETP 33:405409

35. Sadek AA, Ushio M, Matsuda F (1990) Effect of rare earth metal oxide additions to tungsten electrodes. Metall Trans A 12A:32213236

36. He ZH (1995) Contribution à l'étude théorique et exprérimentale de l'interaction plasma-cathode dans un arc électrique. $\mathrm{PhD}$ Thesis, Paris VI University, Paris

37. Vacquié S (2000) Sciences et techniques de l'ingénieur, CNRS editions

38. Coulombe S, Meunier J-L (1997) Thermo-field emission: a comparative study. J Phys D Appl Phys 30:776-780

39. Yamamoto K, Tashiro S, Tanaka M (2009) Numerical analysis of current attachment at thermionic cathode for gas tungsten arc at atmospheric pressure. Transactions of Joining and Welding Research Institute 38:1-5

40. Fomenko VS, Podcherniaeva IA (1975) Emission and adsorption properties of substances and materials: Handbook moscow, Atomizdat, In Russian

41. Fridman A, Kennedy LA (2004) Plasma physics and engineering. CRC Press

42. Mehta VK (2005) Principles of electronics. Chand and Co ltd

43. Lide DR (2008) CRC Handbook of chemistry and physics. Boca Raton, CRC Press/Taylor and Francis

44. Langmuir I (1923) The electron emission from tungsten filaments containing thorium. Phys Rev 22:357-398

45. Zhou X, Heberlein J (1998) An experimental investigation of factors affecting arc-cathode erosion. J Phys D Appl Phys 44:505203(15pp)

46. Tanaka M, Ushio M, Ikeuchi M, Kagebayashi Y (2005) In situ measurements of electrode work functions in free-burning arcs during operation at atmospheric pressure. J Phys D Appl Phys 38:29-35

47. Sillero J, Ortega D, Munoz-Serrano E, Casado E (2010) An experimental study of thoriated tungsten cathodes operating at different current intensities in an atmospheric-pressure plasma torch. J Phys D Appl Phys 43:185204(8pp)

48. Benilov MS (1999) Analysis of ionization non-equilibrium in the near-cathode region of atmospheric-pressure arcs. J Phys D Appl Phys 32:257-262 
49. Benilov MS, Cunha MD (2002) Heating of refractory cathodes by high-pressure arc plasmas: I. J Phys D Appl Phys 35:1736-1750

50. Haidar J (1999) Non-equilibrium modelling of transferred arcs. J Phys D Appl Phys 32:263-272

51. Schmitz H, Riemann KU (2001) Consistent analysis of the boundary layer of a Saha plasma. J Phys D Appl Phys 34:1193-1202

52. Nandelstädt D, Redwitz M, Dabringhausen L, Luhmann J, Lichtenberg S, Mentel J (2002) Determination of HID electrode falls in a model lamp III: results and comparison with theory. J Phys D Appl Phys 35:1639-1647

53. Baeva M, Uhrlandt D (2013) Plasma chemistry in the free-burning Ar arc. J Phys D Appl Phys 46:325202(9pp)

54. Katsonis K (1976) Etude statistique et cinétique de plasmas d'argon en dehors de l'équilibre thermodynamique local. $\mathrm{PhD}$ Thesis, Paris XI University, Orsay

55. Beulens JJ, Milojevic D, Schram DC, Vallinga PM (1991) A twodimensional nonequilibrium model of cascaded arc plasma flows. Phys Fluids B 3:2548-2557

56. Mitchner M, Kruger CH (1973) Partially ionized gases. Wiley, New York

57. Fridman A, Kennedy LA (2011) Plasma physics and engineering, CRC press, 2nd edn

58. Hartung LC (1991) Nonequilibrium radiative heating prediction model for aeroassisted flowfields with coupling to flowfield solvers. PhD Thesis North Carolina State University, Raleigh

59. Hahn Y (1997) Electron-ion recombination processes-an overview. Rep Prog Phys 60:691-759

60. Schmidt J, Upadhyay PP, Herdrich G, Bauder U (2015) Review of reaction rate coefficients of non-equilibrium argon for electron impact ionization. In: 8th European Symposium on Aerothermodynamics for space vehicles, Lisbon

61. Hinnov E, Hirschberg JG (1962) Electron-ion recombination in dense plasmas. Phys Rev 125(3):795-801

62. Rat V, Murphy AB, Aubreton J, Elchinger M-F, Fauchais P (2008) Treatment of non-equilibrium phenomena in thermal plasma flows. J Phys D Appl Phys 41:183001(28pp)

63. Dabringhausen L, Langenscheidt O, Lichtenberg S, Redwitz M, Mentel J (2005) Different modes of arc attachment at HID cathodes: simulation and comparison with measurements. J Phys D Appl Phys 38:3128-3142

64. Schnick M, Dreher M, Zschetzsche J, Fuessel U, Spille-Kohoff A (2012) Visualization and optimization of shielding gas flows in arc welding. Welding in the World 56(1-2):54-61

65. Cressault Y, Rou ME, Gleizes A, Meillot E (2010) Net emission of Ar- $\mathrm{H}_{2}-\mathrm{He}$ thermal plasmas at atmospheric pressure. J Phys D Appl Phys 43:335204(13pp)

66. Cressault Y, Gleizes A (2013) Thermal plasma properties for Ar$\mathrm{Al}, \mathrm{Ar}-\mathrm{Fe}$ and $\mathrm{Ar}-\mathrm{Cu}$ mixtures used in welding plasmas processes: I. Net emission coefficients at atmospheric pressure. J Phys D Appl Phys 46:415206(16pp)
67. Sevastayanenko VG, Bakken JA (1998) Radiative transfer in industrial thermal plasmas of complex composition. In: 5th european conference on thermal plasma processes (TPP-5), St. Petersburg

68. Zollweg RJ, Lowke JJ, Liebermann RW (1975) Arc constriction in lamps containing mercury and iodine. J Appl Phys 46: 3828-3838

69. Kovitya P, Lowke JJ (1985) Two-dimensional analysis of freeburning arcs in argon. J Phys D Appl Phys 18:53-70

70. Haidar J (1995) Local thermodynamic equilibrium in the cathode region of a free burning arc in argon. J Phys D Appl Phys 28:24942504

71. Choquet I, Javidi Shirvan A, Nilsson H (2012) On the choice of electromagnetic model for short high-intensity arcs, applied to welding. J Phys D Appl Phys 45:205203(14pp)

72. Freton P, Gonzalez J-J, Ranarijaona Z, Mougenot J (2012) Energy equation formulations for two-temperature modelling of thermal plasmas. J Phys D Appl Phys 45:465206(22pp)

73. Rat V, André $P$, Aubreton J, Elchinger MF, Fauchais $P$, Vacher D (2002) Transport coefficients including diffusion in a two-temperature argon plasma. J Phys D Appl Phys 35:981991

74. Delalondre C (1990) Modélisation aérothermodynamique d'arcs électriques à forte intensité avec prise en compte du déséquilibre thermodynamique local et du transfert thermique à la cathode. $\mathrm{PhD}$ Thesis, Rouen University, Rouen

75. Rat V, Pascal A, Aubreton J, Elchinger MF, Fauchais P, Lefort A (2001) Transport properties in a two-temperature plasma: theory and application. Phys Rev E 64:026409(20pp)

76. Van de Sanden MCM, Schram P, Peeters AG, Van der Mullen JAM, Kroesen GMW (1989) Thermodynamic generalization of the Saha equation for a two-temperature plasma. Phys Rev A 40:5273-5276

77. Kramid A, Ralchenko Y, Reader J, NIST ASD Team (2013) NIST atomic spectra database (ver. 5.0). http://physics.nist.gov/asd

78. Gleizes A, Chevry B, Gonzales J-J (1999) Calculation of a twotemperature plasma composition: bases and application to SF6. J Phys D Appl Phys 32:2060-2067

79. Kunze HJ (2009) Introduction to plasma spectroscopy, Springer series on Atomic, Optical, and Plasma Physics, Springer

80. Morrow R, Lowke J (1993) A one-dimensional theory for the electrode sheaths of electric arcs. J Phys D Appl Phys 26:634642

81. Goodarziy M, Chooz R, Toguri JM (1997) The effect of the cathode tip angle on the GTAW arc and weld pool: I. Mathematical model of the arc. J Phys D Appl Phys 30:2744-2756

82. Lago F, Gonzalez JJ, Freton P, Gleizes A (2004) A numerical modelling of an electric arc and its interaction with the anode: Part I. The 3D model. J Phys D Appl Phys 37:883-897

83. Yih SW, Wang CT (1979) Tungsten: sources, metallurgy, properties, and applications. Plenum publishing corporation 